Document downloaded from:

http://hdl.handle.net/10251/102246

This paper must be cited as:

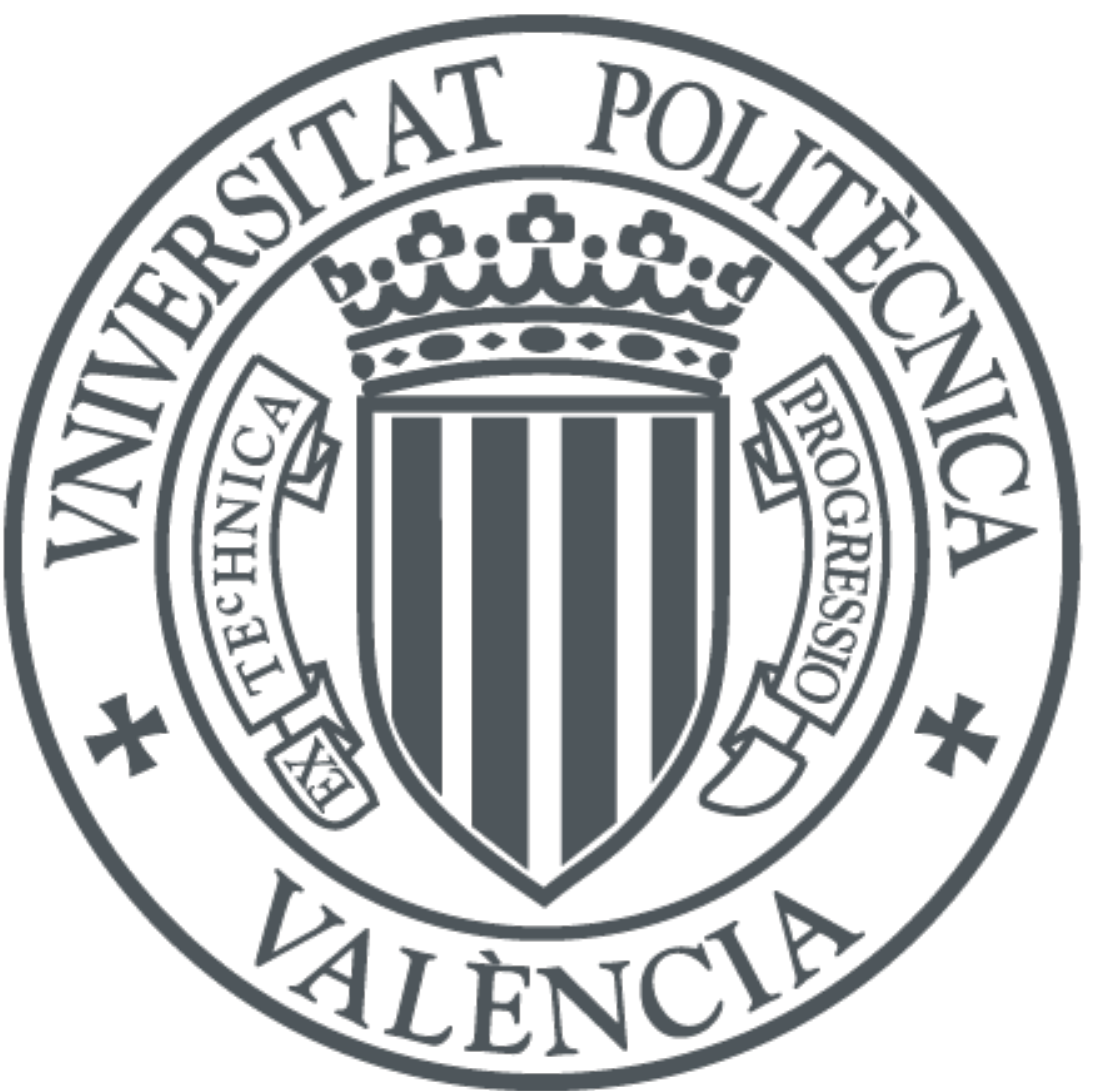

The final publication is available at

https://doi.org/10.1007/s11071-016-3089-2

Copyright Springer-Verlag

Additional Information 


\title{
Widening basins of attraction of optimal iterative methods
}

\author{
Parisa Bakhtiari • Alicia Cordero - Taher Lotfi • \\ Kathayoun Mahdiani • Juan R. Torregrosa
}

Received: date / Accepted: date

\begin{abstract}
In this work, we analyze the dynamical behavior on quadratic polynomials of a class of derivativefree optimal parametric iterative methods, designed by Khattri and Steihaug. By using their parameter as an accelerator, we develop different methods with memory of orders three, six and twelve, without adding new functional evaluations. Then a dynamical approach is made, comparing each of the proposed methods with the original ones without memory, with the following empiric conclusion: basins of attraction of iterative schemes with memory are wider and the behavior is more stable. This has been numerically checked by estimating the solution of a practical problem, as the friction factor of a pipe and also of other nonlinear academic problems.
\end{abstract}

Keywords Multi-point iterative methods · dynamical plane · basin of attraction · with and without memory methods · Kung and Traub's conjecture · efficiency index

\section{Introduction}

Nonlinear equations $f(x)=0$ usually appear when a modelization of a real problem is made. The estimation of one of its solutions $\alpha$ by means of iterative techniques is often used, starting from an appropriate initial guess. Indeed, in the last years a dynamical approach of these kind of methods is usually made in order to analyze their stability. The most used techniques are the complex analysis of the fixed and critical points of the rational operator associated to the studied iterative method applied on a simple polynomial of low degree. Conditions that assure a stable or unstable behavior of the rational fixed point operator will yield to a similar behavior of the scheme on more complex functions, as if a numerical scheme has a bad behavior on a simple polynomial, it is quite probable to hold this performance on other functions. This kind of analysis has been made recently

This research was supported by Islamic Azad University, Hamedan Branch, Ministerio de Economía y Competitividad MTM201452016-C02-2-P and Generalitat Valenciana PROMETEO/2016/089.

\section{Parisa Bakhtiari}

Young Researchers and Elite Club, Hamedan Branch,

Islamic Azad University, Hamedan, Iran

E-mail: bakhtiari@iauh.ac.ir

Alicia Cordero

Instituto Universitario de Matemática Multidisciplinar

Universitat Politècnica de València, Spain

E-mail: acordero@mat.upv.es

Taher Lotfi

Department of Mathematics, Hamedan Branch, Islamic Azad University, Hamedan, Iran

E-mail: lotfi@iauh.ac.ir

Kathayoun Mahdiani

University, Hamedan, Iran

E-mail: mahdiani@iauh.ac.ir

Juan R.Torregrosa

Instituto Universitario de Matemática Multidisciplinar

Universitat Politècnica de València, Spain

E-mail: jrtorre@mat.upv.es 
by different authors, as Amat et al. in [1,2], Chun et al. in [3], Babajee et al. in [4] or Magreñan et al. in [5] (among others) and has proved to be very useful to analyze the reliability and stability of the designed iterative methods.

Under the numerical points of view, there exist numerous variants of Newton' and Steffensen's methods, improving their order of convergence, developed in the last years. We can see an interesting overview of these variants in [6]. Ostrowski in [7] defined an index for comparing iterative methods, by means of the order of convergence $p$ and the number of functional evaluations per iteration, $d$, as $I=p^{\frac{1}{d}}$. Kung and Traub conjectured in [8] that the order of convergence of an iterative method without memory with $d$ functional evaluations per step is, at most, $2^{d-1}$. When the order of convergence of a method reaches this bound, it is called optimal.

For many practical problems, to calculate the derivatives of the nonlinear function to be solved is a hard task, or even impossible if it is not known explicitly. This fact induced the design of derivative-free iterative methods of high order of convergence. In fact, there exist in the literature some classes of optimal schemes of arbitrary order of convergence, such us the one designed by Kung and Traub in [8] and by Khattri and Steihaug in [9]. In fact, the last work is the starting point of this manuscript.

In [9], the authors design a class of optimal one-parametric derivative-free iterative methods of arbitrary order, starting from a modified Steffensen's method in which $w_{n}=x_{n}+\gamma f\left(x_{n}\right), \gamma \in \mathbb{R}, \gamma \neq 0$. This scheme is obtained by substituting the derivative of Newton's scheme by $f^{\prime}\left(x_{n}\right) \approx \eta_{1} f\left(x_{n}\right)+\eta_{2} f\left(w_{n}\right)$. Assuming that this approximation is true for $f(t)=1$ and $f(t)=t$, they find $\eta_{2}=-\eta_{1}=\frac{1}{\gamma f^{\prime}(\alpha)}$. Therefore, $f^{\prime}\left(x_{n}\right) \approx$ $\frac{f\left(w_{n}\right)-f\left(x_{n}\right)}{\gamma f\left(x_{n}\right)}=f\left[x_{n}, w_{n}\right]$. Let us note that the dumping parameter plays an important role in the subsequent extension to methods with memory. The error equation of this iterative scheme is

$$
e_{n+1}=A_{1}\left(1+\gamma f^{\prime}(\alpha)\right) e_{n}^{2}+O\left(e_{n}^{3}\right)
$$

where $A_{1}=\frac{c_{2}}{c_{1}}$, being $c_{j}=\frac{1}{j !} f^{(j)}(\alpha), j=1,2, \ldots$ So, the second order of convergence does not depend on the value of the parameter $\gamma$.

By using the same procedure, the authors of [9] obtained a family of optimal two-point methods without memory, whose iterative expression is

$$
\left\{\begin{array}{l}
y_{n}=x_{n}-\gamma \frac{f\left(x_{n}\right)^{2}}{f\left(w_{n}\right)-f\left(x_{n}\right)}, \\
x_{n+1}=y_{n}-\frac{f\left(y_{n}\right)}{\frac{w_{n}-y_{n}}{\left(x_{n}-y_{n}\right) \gamma}-\frac{\left(x_{n}-y_{n}\right)}{\left(w_{n}-y_{n}\right) \gamma f\left(x_{n}\right)} f\left(w_{n}\right)-\frac{w_{n}+x_{n}-2 y_{n}}{\left(x_{n}-y_{n}\right)\left(w_{n}-y_{n}\right)} f\left(y_{n}\right)}, n=0,1,2, \ldots
\end{array}\right.
$$

Khattri and Steihaug proved the optimal fourth-order of convergence of this family, that we will denote by M4, whose error equation is

$$
e_{n+1}=A_{2}\left(1+\gamma f^{\prime}(\alpha)\right)^{2} e_{n}^{4}+O\left(e_{n}^{5}\right)
$$

where $A_{2}=\frac{c_{2}}{c_{1}^{3}}\left(c_{2}^{2}-c_{1} c_{3}\right)$.

Finally, by adding a new step, they obtain the following family of optimal three-point methods

$$
\left\{\begin{array}{l}
y_{n}=x_{n}-\gamma \frac{f\left(x_{n}\right)^{2}}{f\left(w_{n}\right)-f\left(x_{n}\right)}, \\
z_{n}=y_{n}-\frac{f\left(y_{n}\right)}{\frac{w_{n}-y_{n}}{\left(x_{n}-y_{n}\right) \gamma}-\frac{\left(x_{n}-y_{n}\right)}{\left(w_{n}-y_{n}\right) \gamma f\left(x_{n}\right)} f\left(w_{n}\right)-\frac{w_{n}+x_{n}-2 y_{n}}{\left(x_{n}-y_{n}\right)\left(w_{n}-y_{n}\right)} f\left(y_{n}\right)}, \\
x_{n+1}=z_{n}-\frac{f\left(z_{n}\right)}{H_{1}+H_{2}+H_{3}+H_{4}}, n=0,1,2, \ldots,
\end{array}\right.
$$


where

$$
\begin{aligned}
H_{1} & =-\frac{\left(y_{n}-z_{n}\right)\left(w_{n}-z_{n}\right)}{\left(x_{n}-z_{n}\right) \gamma\left(x_{n}-y_{n}\right)}, \\
H_{2} & =\frac{\left(y_{n}-z_{n}\right)\left(x_{n}-z_{n}\right)}{\left.\left(w_{n}-z_{n}\right)\left(w_{n}-y_{n}\right)\left(w_{n}-x_{n}\right)\right)} f\left(w_{n}\right), \\
H_{3} & =\frac{w_{n} x_{n}-w_{n} z_{n}-x_{n} z_{n}+z_{n}^{2}}{\left(y_{n}-w_{n}\right)\left(y_{n}-x_{n}\right)\left(y_{n}-z_{n}\right)} f\left(y_{n}\right), \\
H_{4} & =\frac{w_{n} x_{n}+w_{n} y_{n}-2 w_{n} z_{n}+x_{n} y_{n}-2 x_{n} z_{n}-2 y_{n} z_{n}+3 z_{n}^{2}}{\left(z_{n}-w_{n}\right)\left(z_{n}-x_{n}\right)\left(z_{n}-y_{n}\right)} f\left(z_{n}\right) .
\end{aligned}
$$

We will denote this class by M8. Regarding its error equation, let us notice that it is mistaken in the original manuscript, although the order of the method is correct. The accurate one is the following:

$$
e_{n+1}=A_{3}\left(1+\gamma f^{\prime}(\alpha)\right)^{4} e_{n}^{8}+O\left(e_{n}^{9}\right)
$$

where $A_{3}=\frac{c_{2}^{2}}{c_{1}^{7}}\left(c_{2}^{2}-c_{1} c_{3}\right)\left(c_{2}^{3}-c_{1} c_{3} c_{2}+c_{1}^{2} c_{4}\right)$.

Taking into account the error equations of the methods, we conjecture that the corresponding error equation of an optimal multipoint iterative class constructed in the same way with $k$ steps, is given by

$$
e_{n+1}=A_{k}\left(1+\gamma f^{\prime}(\alpha)\right)^{2^{k-1}} e_{n}^{2^{k}}
$$

where $A_{k}$ is a constant that depends on $c_{m}, m=1,2, \ldots, k+1$. It differs from the original general error equation proposed by Khattri and Steihaug in the exponent of the power of $\left(1+\gamma f^{\prime}(\alpha)\right)$, which was $k$.

We will use in the following sections the symbols $\rightarrow, O$ and $\sim$ according to the conventions appearing in [10]: If $\lim _{n \rightarrow \infty} g\left(x_{n}\right)=C$, we write $g\left(x_{n}\right) \rightarrow C$ or $g \rightarrow C$. If $\lim _{x \rightarrow \alpha} g(x)=C$, we write $g(x) \rightarrow C$ or $g \rightarrow C$. If $\frac{f}{g} \rightarrow C$, where $C$ is a nonzero constant, we write $f=O(g), f \sim C g$ or $f \sim g$, for the sake of simplicity and without loss of generality.

In order to study the dynamical behavior of the iterative methods M4 and M8 (the second-order scheme is Steffensen's method and its damped version was studied in [11] and the references therein), it is necessary to recall some basic dynamical concepts. For a more extensive review of these concepts, see [12].

Let $R: \hat{\mathbb{C}} \rightarrow \hat{\mathbb{C}}$ be an operator that results from applying an iterative method on a particular function, where $\hat{\mathbb{C}}$ is the Riemann sphere. The orbit of a point $z_{0} \in \hat{\mathbb{C}}$ is defined as the set of successive images of $z_{0}$ by the operator, $\left\{z_{0}, R\left(z_{0}\right), \ldots, R^{n}\left(z_{0}\right), \ldots\right\}$.

The orbit of a point on the complex plane can be classified depending on its asymptotic behavior. In this way, a point $z_{0} \in \mathbb{C}$ is a fixed point of $R$ if $R\left(z_{0}\right)=z_{0}$. A fixed point is attracting, repelling or neutral (parabolic) if $\left|R^{\prime}\left(z_{0}\right)\right|$ is lower than, greater than or equal to 1 , respectively. Moreover, if $\left|R^{\prime}\left(z_{0}\right)\right|=0$, the fixed point is superattracting.

If $z_{f}^{*}$ is an attracting fixed point of operator $R$, its basin of attraction $\mathcal{A}\left(z_{f}^{*}\right)$ is defined as the set of pre-images of any order such that

$$
\mathcal{A}\left(z_{f}^{*}\right)=\left\{z_{0} \in \hat{\mathbb{C}}: R^{n}\left(z_{0}\right) \rightarrow z_{f}^{*}, n \rightarrow \infty\right\} .
$$

The set of points whose orbits tends to any attracting periodic point $z_{f}^{*}$ is defined as the Fatou set, $\mathcal{F}(R)$. The complementary set, the Julia set $\mathcal{J}(R)$, is the closure of the set of pre-images of its repelling fixed points, and establishes the boundaries between the basins of attraction.

The rest of the paper is organized as follows: in Section 2, we analyze the dynamical behavior of classes M4 and M8 on quadratic polynomials, showing the stability of the methods in terms of the value of parameter $\gamma$. In Section 3, some iterative methods with memory are developed and their convergence is analyzed. Moreover, in Section 4 a comparative study between the basins of convergence associated to methods with and without memory is made. Section 5 is devoted to analyze the numerical performance of the proposed methods with memory respect their original optimal schemes. To get this aim, the calculation of the friction factor of a pipe by solving the Colebrook-White equation is made and also some academic nonlinear problems are under study, where proposed schemes are compared with other methods with memory. Finally, some concluding remarks are presented. 


\section{Dynamical analysis of methods without memory}

Our aim in this section is to study the general convergence of families M4 and M8 for quadratic polynomials. To be more precise (see [13]), a given method is generally convergent if the scheme converges to a root for almost every starting point and for almost every polynomial of a given degree. As no Scaling Theorem can be stated for derivative-free methods (see, for example [14]), only the behavior on specific polynomials can be analyzed. We will use the polynomial $p(z)=z^{2}-1$ for this purpose. Then, let us denote by $O_{M 4}(z, \gamma)\left(O_{M 8}(z, \gamma)\right)$ the operator associated to the fourth-order (resp. eighth-order) scheme on $p(z)$. Although this study is made only for quadratic polynomials, the results can be extrapolated to any nonlinear function in the following sense: if a method (corresponding to an specific value of the parameter) has bad behavior on a simple low-degree polynomial, it is quite probable that this behavior holds in more complicated functions. This will be checked in the numerical section of this manuscript. This is a standard procedure used in last years for many authors in this area of research (see, for example $[2,5,15,16,17])$.

\subsection{Dynamical behavior of M4}

The rational function obtained when M4 is applied on $p(z)$ is

$$
O_{M 4}(z, \gamma)=\frac{\left(-1+z^{2}\right)\left(16 \gamma^{2} z^{3}+2 \gamma^{4} z\left(-1+z^{2}\right)^{2}+\gamma^{3}\left(-1+z^{2}\right)\left(1+9 z^{2}\right)\right)+4\left(z+z^{3}\right)+\gamma\left(-3-2 z^{2}+13 z^{4}\right)}{2\left(z+\gamma\left(-1+z^{2}\right)\right)\left(2 z+\gamma\left(-1+z^{2}\right)\right)\left(2+2 \gamma z+\gamma^{2}\left(-1+z^{2}\right)\right)}
$$

It is known that the fixed points of $O_{M 4}(z, \gamma)$ are the roots of the equation $O_{M 4}(z, \gamma)=z$, that is, the roots of $p(z), z=-1, z=1$ and the so-called strange fixed points. In the following result these fixed points are described.

Proposition 1 The fixed points of operator $O_{M 4}(z, \gamma)$ are the roots of $p(z)$ and, if $\gamma \neq-1$ and $\gamma \neq 1$, five strange fixed points:

$-z=\infty$, (divergent behavior) whose character is parabolic.

- Four points depending on parameter $\gamma$ :

$$
\begin{aligned}
s_{1}(\gamma)= & -\frac{1}{6 \gamma}\left(6+\sqrt{3} \gamma \sqrt{1 / \gamma^{3}\left(19 \gamma-2 \gamma^{3}+3 \gamma\left(-7+2 \gamma^{2}\right)+\frac{\gamma^{2}-16 \gamma^{4}+16 \gamma^{6}}{A(\gamma)}+A(\gamma)\right)}\right. \\
& +\sqrt{3} \gamma \sqrt{\left.8-\frac{4}{\gamma^{2}}+\frac{-1+16 \gamma^{2}-16 \gamma^{4}}{\gamma A(\gamma)}-\frac{A(\gamma)}{\gamma^{3}}-\frac{12 \sqrt{3}}{\gamma^{3} B(\gamma)}\right)}, \\
s_{2}(\gamma)= & \frac{1}{6 \gamma}\left(-6-\sqrt{3} \gamma \sqrt{1 / \gamma^{3}\left(19 \gamma-2 \gamma^{3}+3 \gamma\left(-7+2 \gamma^{2}\right)+\frac{\gamma^{2}-16 \gamma^{4}+16 \gamma^{6}}{A(\gamma)}+A(\gamma)\right)}\right. \\
& \left.+\sqrt{3} \gamma \sqrt{8-\frac{4}{\gamma^{2}}+\frac{-1+16 \gamma^{2}-16 \gamma^{4}}{\gamma A(\gamma)}-\frac{A(\gamma)}{\gamma^{3}}-\frac{12 \sqrt{3}}{\gamma^{3} B(\gamma)}}\right), \\
s_{3}(\gamma)= & \frac{1}{6 \gamma}\left(-6+\sqrt{3} \gamma \sqrt{1 / \gamma^{3}\left(19 \gamma-2 \gamma^{3}-3 \gamma\left(-7+2 \gamma^{2}\right)+\frac{\gamma^{2}-16 \gamma^{4}+16 \gamma^{6}}{A(\gamma)}+A(\gamma)\right)}\right. \\
& \left.+\sqrt{3} \gamma \sqrt{8-\frac{4}{\gamma^{2}}+\frac{-1+16 \gamma^{2}-16 \gamma^{4}}{\gamma A(\gamma)}-\frac{A(\gamma)}{\gamma^{3}}+\frac{12 \sqrt{3}}{\gamma^{3} B(\gamma)}}\right), \\
s_{4}(\gamma)= & \frac{1}{6 \gamma}\left(-6+\sqrt{3} \gamma \sqrt{1 / \gamma^{3}\left(19 \gamma-2 \gamma^{3}+3 \gamma\left(-7+2 \gamma^{2}\right)+\frac{\gamma^{2}-16 \gamma^{4}+16 \gamma^{6}}{A(\gamma)}+A(\gamma)\right)}\right. \\
& \left.+\sqrt{3} \gamma \sqrt{8-\frac{4}{\gamma^{2}}+\frac{-1+16 \gamma^{2}-16 \gamma^{4}}{\gamma A(\gamma)}-\frac{A(\gamma)}{\gamma^{3}}+\frac{12 \sqrt{3}}{\gamma^{3} B(\gamma)}}\right),
\end{aligned}
$$

where

$$
A(\gamma)=\sqrt[3]{55 \gamma^{3}+30 \gamma^{5}-96 \gamma^{7}+64 \gamma^{9}+6 \sqrt{3} \sqrt{\gamma^{6}\left(28+31 \gamma^{2}-97 \gamma^{4}+64 \gamma^{6}\right)}}
$$

and

$$
B(\gamma)=\sqrt{\frac{1}{\gamma^{3}}\left(19 \gamma-2 \gamma^{3}+3 \gamma\left(-7+2 \gamma^{2}\right)+\frac{\gamma^{2}-16 \gamma^{4}+16 \gamma^{6}}{A(\gamma)}+A(\gamma)\right)}
$$


Moreover, $s_{2}(\gamma)=s_{3}(\gamma)$ if $\gamma \in\{1.00751 \pm 0.268746 i,-1.00751 \pm 0.268746 i, \pm 0.608333 i\}$, where they are neutral. In general, the strange fixed points can be attractive, or even superattractive, in small areas of the complex region $[-2,2] \times[-2,2]$ (see Figure 1).

On the other hand, if $\gamma=-1$ or $\gamma=1$, fixed point operator is simplified,

$$
\begin{aligned}
O_{M 4}(z,-1) & =\frac{2+6 z+5 z^{2}-2 z^{3}-5 z^{4}+2 z^{5}}{2\left(-1-2 z+z^{2}\right)\left(-1-z+z^{2}\right)} \\
O_{M 4}(z, 1) & =\frac{-2+6 z-5 z^{2}-2 z^{3}+5 z^{4}+2 z^{5}}{2\left(-1+z+z^{2}\right)\left(-1+2 z+z^{2}\right)},
\end{aligned}
$$

and the fixed points are, respectively,

$$
\begin{aligned}
& \left\{-1,1-\frac{1}{(27-3 \sqrt{78})^{1 / 3}}-\frac{(9-\sqrt{78})^{1 / 3}}{3^{2 / 3}}, 1+\frac{(1 \pm i \sqrt{3})(27-3 \sqrt{78})^{1 / 3}}{6}+\frac{3^{1 / 6}(\mp 3 i+\sqrt{3})}{6(9-\sqrt{78})^{1 / 3}}\right\} \\
& \left\{1,-1+\frac{(27-3 \sqrt{78})^{1 / 3}+(27+3 \sqrt{78})^{1 / 3}}{3},-1-\frac{(1+i \sqrt{3})(27 \mp 3 \sqrt{78})^{1 / 3}}{6}+i \frac{(i+\sqrt{3})(27 \pm 3 \sqrt{78})^{1 / 3}}{6}\right\},
\end{aligned}
$$

that is, the root $z=1$ is not a fixed point of $O_{M 4}(z,-1)$ and $z=-1$ is not a fixed point of $O_{M 4}(z, 1)$. The strange fixed points are, in both cases, repulsive.

Let us remark the fact that usual values of the parameter as $\gamma= \pm 1$ yield the disappearance of one of the roots of the polynomials as a fixed point of the iterative method.

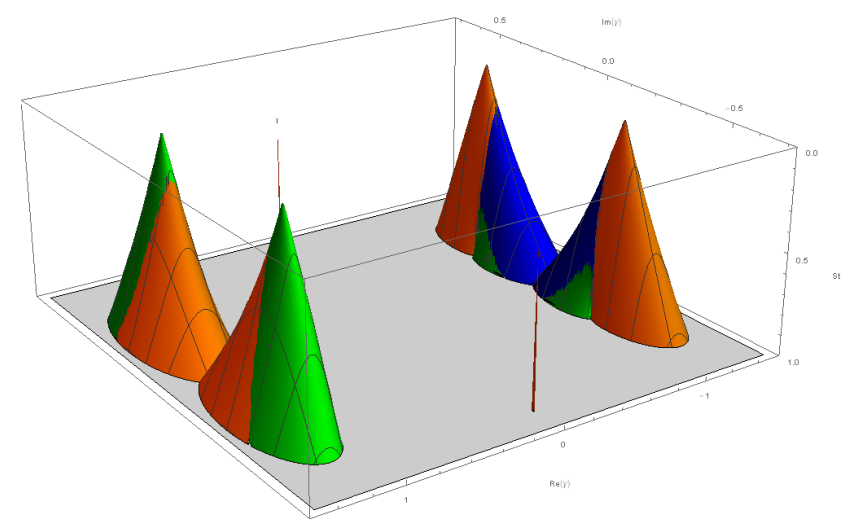

(a) $S t_{s_{i}}(\gamma), i=1,2,3,4$

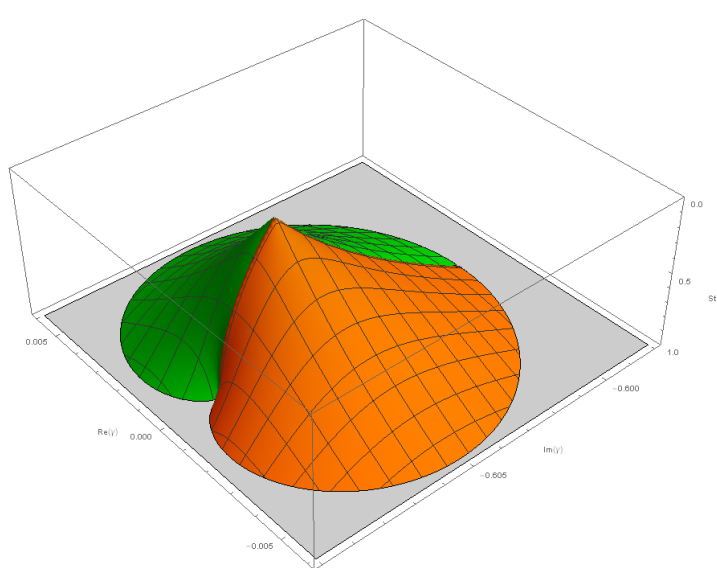

(b) A detail

Fig. 1: Stability function of attractive strange fixed points of M4

The mentioned stability of the strange fixed points can be deduced from numerical and graphical analysis of the respective stability function $S t_{s_{i}}(\gamma)=\left|O_{M 4}^{\prime}\left(s_{i}, \gamma\right)\right|, i=1,2,3,4$; that is, studying the regions of the complex plane in which the absolute value of the derivative of the operator evaluated at the strange fixed point is lower than one. From the numerical analysis of these stability functions, it can be deduced that

i) $s_{1}(\gamma)$ and $s_{4}(\gamma)$ can be attracting but never superattracting.

ii) $s_{2}(\gamma)$ is superattracting if $\gamma=-1.12945 \pm 0.30315 i$ or $\gamma=0.606291 i$.

iii) $s_{3}(\gamma)$ is superattracting if $\gamma=1.12945 \pm 0.30315 i$ or $\gamma=-0.606291 i$. 
Then, the region of the complex plane where these four fixed points can be attractive is represented in Figure 1. The values of the parameter that yield to any strange fixed point to be stable is painted in different colors: it is red, orange, green or blue if $s_{1}(\gamma), s_{2}(\gamma), s_{3}(\gamma)$ or $s_{4}(\gamma)$ is respectively attracting.

It can be observed that there is a stable region around the origin and the unstable behavior is bounded in a corona of radius $r_{1}=0.5$ and $r_{2}=1.5$ around the origin. Out of this corona, no strange fixed point is attracting and the methods can only converge to the roots of the polynomial.

In order to determine the critical points, we calculate the first derivative of $O_{M 4}(z)$. A classical result establishes that there is at least one critical point associated with each invariant Fatou component [12]. It is clear that $z=-1$ and $z=1$ are critical points and give rise to their respective Fatou components (when $\gamma \neq \pm 1$ ), but there exist in the family some free critical points, that is, critical points different from the roots, some of them depending on the value of the parameter. If any of these free critical points is near an stable strange periodic point, then the last one would have its own basin of attraction. This corresponds to a rich dynamical behavior, but also to a bad member of the family under the numerical point of view.
(a) $\gamma=-1$
(b) $\gamma=1$

Fig. 2: Dynamical behavior of M4 on $p(z)$ for $\gamma= \pm 1$
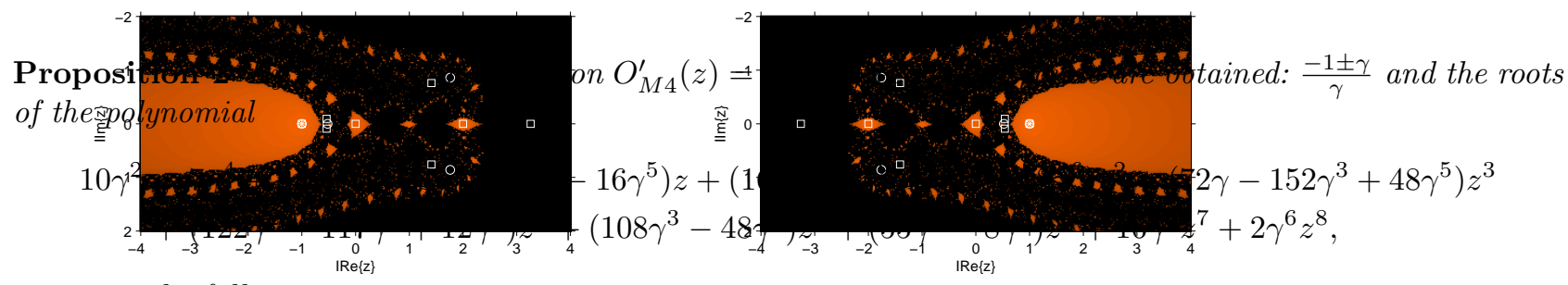

except in the following cases:

- If $\gamma=1$, then there exist seven free critical points: $z=0, z=-2$ and the roots of $5-11 z-5 z^{2}+11 z^{3}+$ $10 z^{4}+2 z^{5}$.

- If $\gamma=-1$, then there are also seven free critical points: $z=0, z=2$ and the roots of $-5-11 z+5 z^{2}+$ $11 z^{3}-10 z^{4}+2 z^{5}$.

- When $\gamma=\frac{1}{2}$, there exist eight free critical points, $z=-3$ and the roots of $67-291 z+137 z^{2}+455 z^{3}+$ $293 z^{4}+91 z^{5}+15 z^{6}+z^{7}$.

- When $\gamma=-\frac{1}{2}$, there also exist eight free critical points, $z=3$ and the roots of $-67-291 z-137 z^{2}+455 z^{3}-$ $293 z^{4}+91 z^{5}-15 z^{6}+z^{7}$.

- If $\gamma=\frac{i}{2}$, then the free critical points are: $z=-1-2 i, z=1-2 i$ and the roots of $-19 i+81 z+126 i z^{2}-$ $50 z^{3}-11 i z^{4}+z^{5}$.

- If $\gamma=-\frac{i}{2}$, then the free critical points are: $z=1+2 i, z=-1+2 i$ and the roots of $19 i+81 z-126 i z^{2}-$ $50 z^{3}+11 i z^{4}+z^{5}$.

In order to visualize the obtained results about values of parameter $\gamma$ associated with changes in the number or stability of the fixed points of operator $O_{M 4}(z)$ or in the number of critical points, we will present different dynamical planes, obtained by using the algorithms designed in [18] and implemented in Matlab. Each one of the figures represents the convergence to the roots of $p(z)$ of the different members of M4. Each point of the plane is a complex initial estimation used to check the behavior of the method. Then, a mesh of $400 \times 400$ points is defined and a point of the plane that presents convergence to the first attracting fixed point is painted in orange (or in green, red, cyan or purple if it converges to the second, third,... fixed point) with a tolerance 


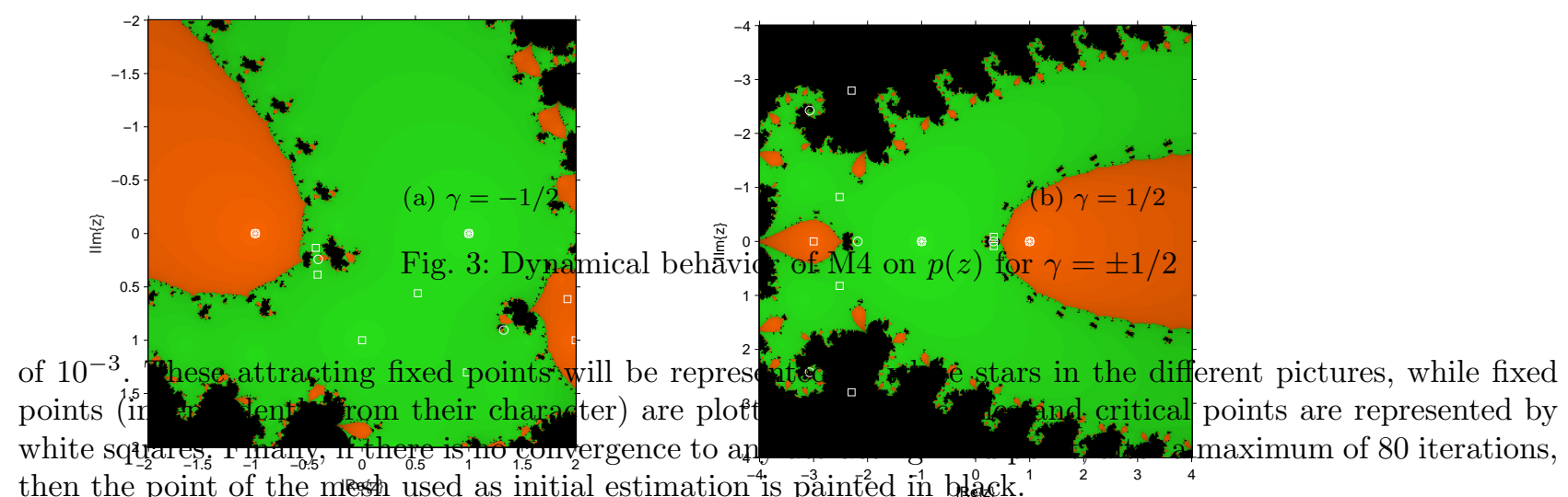
then the point of the mesh used as initial estimation ${ }^{-4}$ is painted in batack. ${ }^{-1}$

Firstly, we present in Figure 2 the dynamical planes associated to $\gamma= \pm 1$; let us remember that, for these values of the parameter, M4 only recognizes one of the roots of $p(z)$ as a fixed point.

For $\gamma$
Figure 3$)$
3
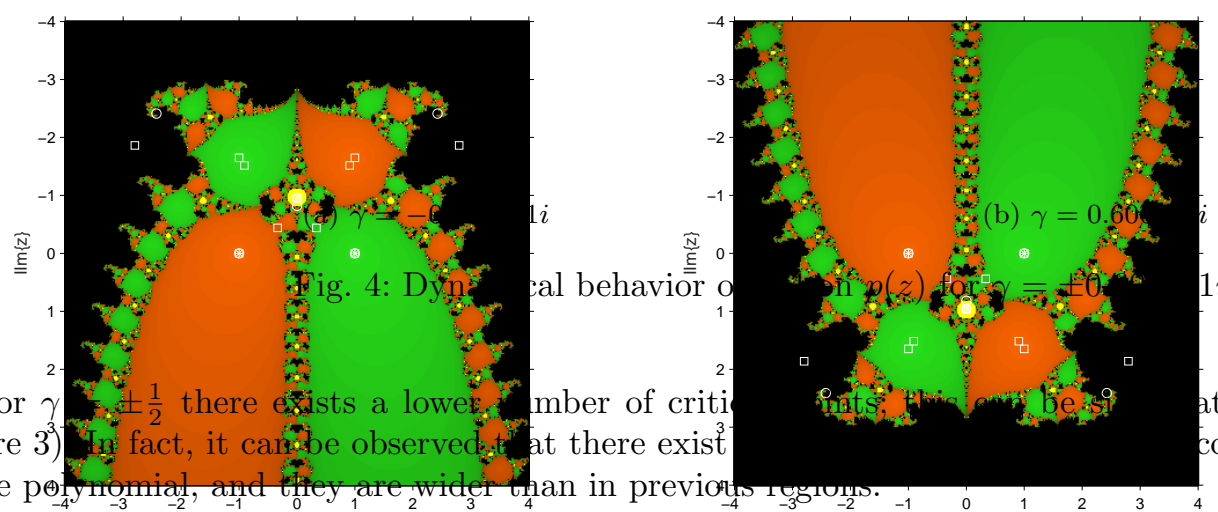

$1 i$

tive for the stability (see corresponding to the roots

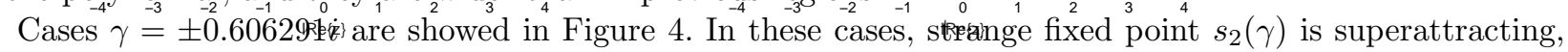
and it has its own basin of attraction (in yellow in the figure). The symmetry of the dynamical plane is a characteristic that will appear again in the following dynamical planes.

When $\gamma=-1.00751 \pm 0.268746 i$ or $\gamma=1.00751 \pm 0.268746 i$, fixed points satisfy $s_{2}(\gamma)=s_{3}(\gamma)$, being both neutral points. In Figure 5 their behavior can be observed. Let us note that, in both cases, there exists a region where these points act as attractors, and one of the orbits of an initial estimation in one of these regions appears in yellow for each dynamical plane. 


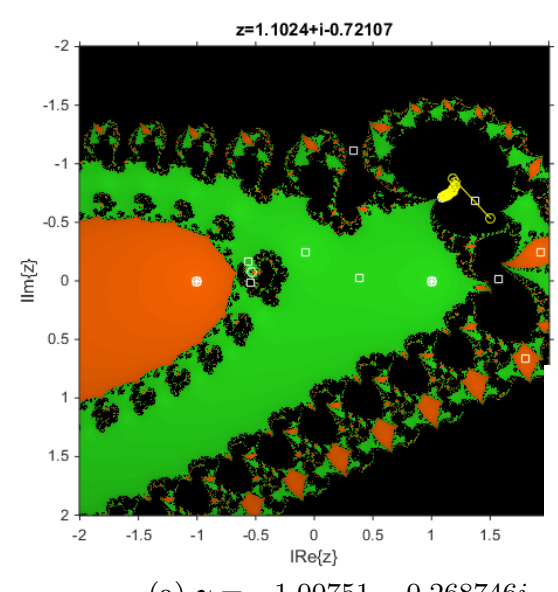

(a) $\gamma=-1.00751-0.268746 i$
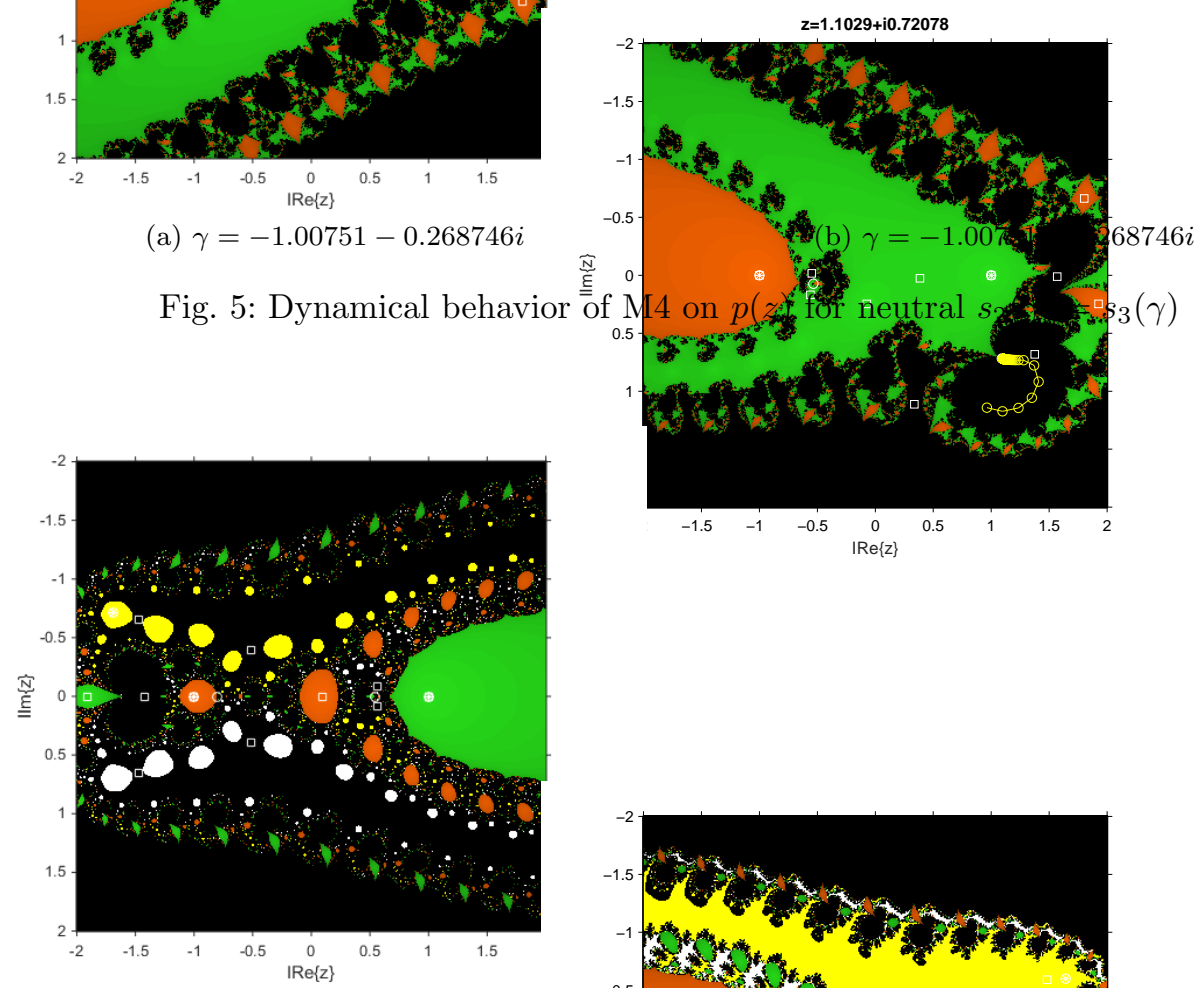

(a) $\gamma=1.1$

Fig. 6: Dynamical behav

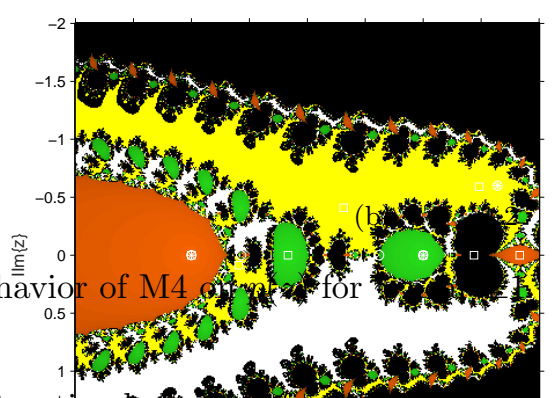

In Figure 6, two dynamical planes showing attractipented, corresponding to real values of the parameter $\gamma$ close to $\gamma= \pm 1^{1.5}$. have been plotted, corresponding to the basins of atta (in yellow and white). lored areas of convergence in $\rightarrow$ green), $s_{2}(\gamma)$ and $s_{4}(\gamma)$

However, the biggest area of the complex plane corresponds to stable behavior of M4, as it is showed in Figure 7, where only two basins of attraction (corresponding to the roots of the polynomial) appear and the rest of fixed points (not attracting ones) appear in Julia set.

\subsection{Dynamical analysis of M8}

Let us consider now the behavior of optimal eighth-order family M8 on polynomial $p(z)$. The associated rational function, denoted by $O_{M 8}(z, \gamma)$, is a quotient of polynomials of degree 14 (numerator) and 13 (denominator), whose coefficients depend on $\gamma$.

The number and stability of the strange fixed points of $O_{M 8}(z, \gamma)$ are described in the following result.

Proposition 3 The number of different strange fixed points of operator $O_{M 8}(z, \gamma)$, for $\gamma \neq \pm 1$, is thirteen, and their stability is described in the following cases: 

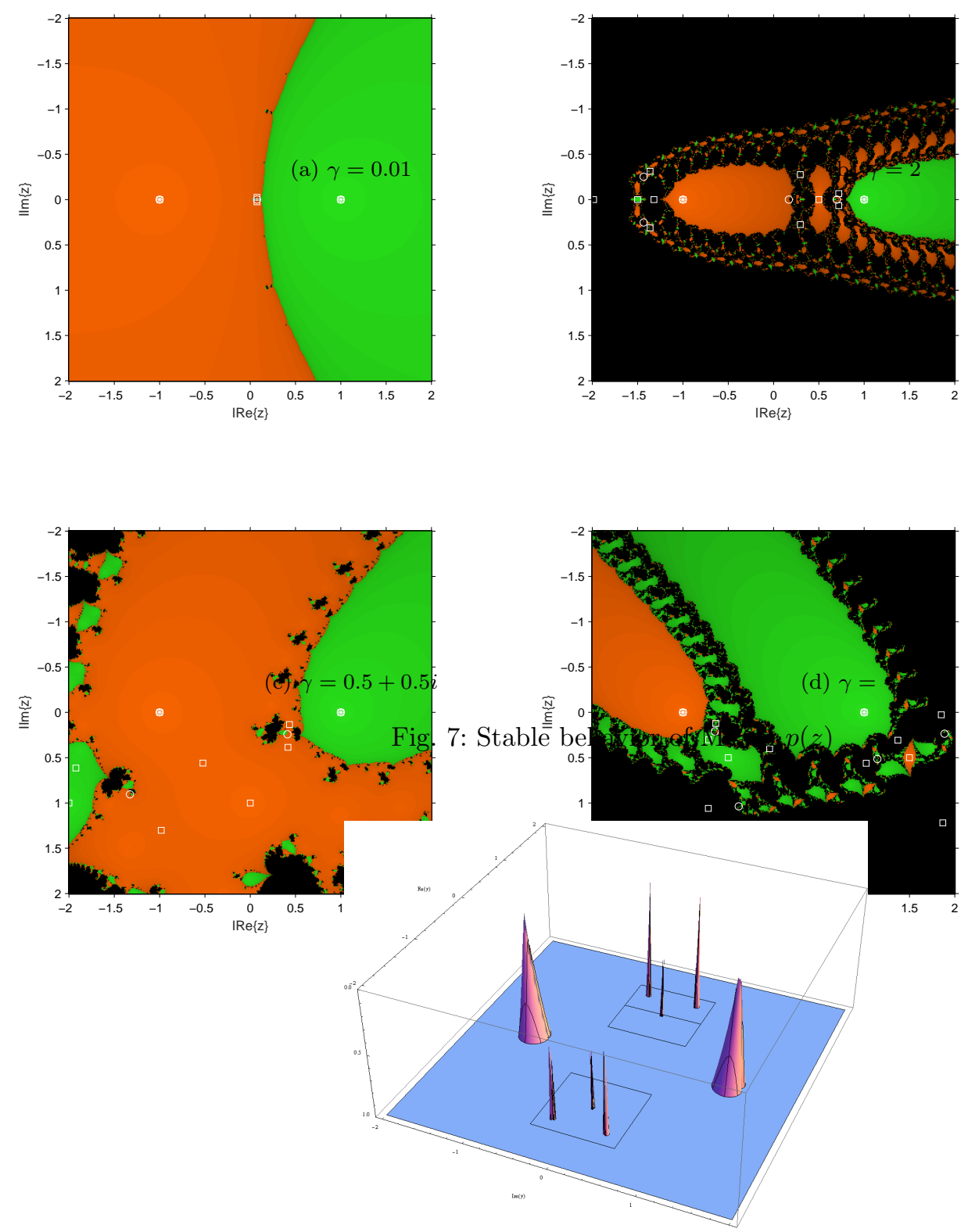

Fig. 8: Stability regions of strange fixed points from M8

i) $z=\infty$ is a fixed point of $O_{M 8}(z, \gamma)$ and it is repulsive, so it remains in the Julia set.

ii) Two of them are always repulsive, so they lay in the Julia set.

iii) The other strange fixed points can be repulsive, attractive or superattractive, in different areas of the complex plane. 
The stability region of each one of these fixed points is represented in Figure 8.

On the other hand, if $\gamma=-1$ or $\gamma=1$, fixed point operator is simplified,

$$
\begin{gathered}
O_{M 8}(z,-1)=\frac{8+48 z+92 z^{2}+28 z^{3}-83 z^{4}-48 z^{5}+14 z^{6}+16 z^{7}+21 z^{8}-20 z^{9}+4 z^{10}}{4\left(-1-2 z+z^{2}\right)\left(-1-z+z^{2}\right)\left(2+6 z+5 z^{2}-2 z^{3}-5 z^{4}+2 z^{5}\right)} \\
O_{M 8}(z, 1)=\frac{8-48 z+92 z^{2}-28 z^{3}-83 z^{4}+48 z^{5}+14 z^{6}-16 z^{7}+21 z^{8}+20 z^{9}+4 z^{10}}{4\left(-1+z+z^{2}\right)\left(-1+2 z+z^{2}\right)\left(-2+6 z-5 z^{2}-2 z^{3}+5 z^{4}+2 z^{5}\right)} .
\end{gathered}
$$

As in case of $M_{4}, z=1$ is not a fixed point of $O_{M 8}(z,-1)$ and $z=-1$ is not a fixed point of $O_{M 8}(z, 1)$. Regarding the strange fixed points, in each case two of them are attracting and the rest are repelling (see Figure g).

By analyzing the stability functions associated to these strange fixed points, it is deduced that there are small regions of the complex plane, inside the corona of radius $r_{1}=0.5$ and $r_{2}=1.5$ where it is easy to find attracting strange fixed points (as, for example, the ones obtained by using $\gamma=1.25-0.4 i$ and $\gamma=0.8 i$, see Figure 10). Moreover, there exist also wide regions of stability out of this corona (see Figure 11).

By analyzing the equation $O_{M 8}^{\prime}(z, \gamma)=0$, we obtain sixteen free critical points, that will coincide with some of the strange fixed points in case these are superattracting. However, there are specific values of parameter $\gamma$ which simplify the fixed point operator and whose number of free critical points is lower.

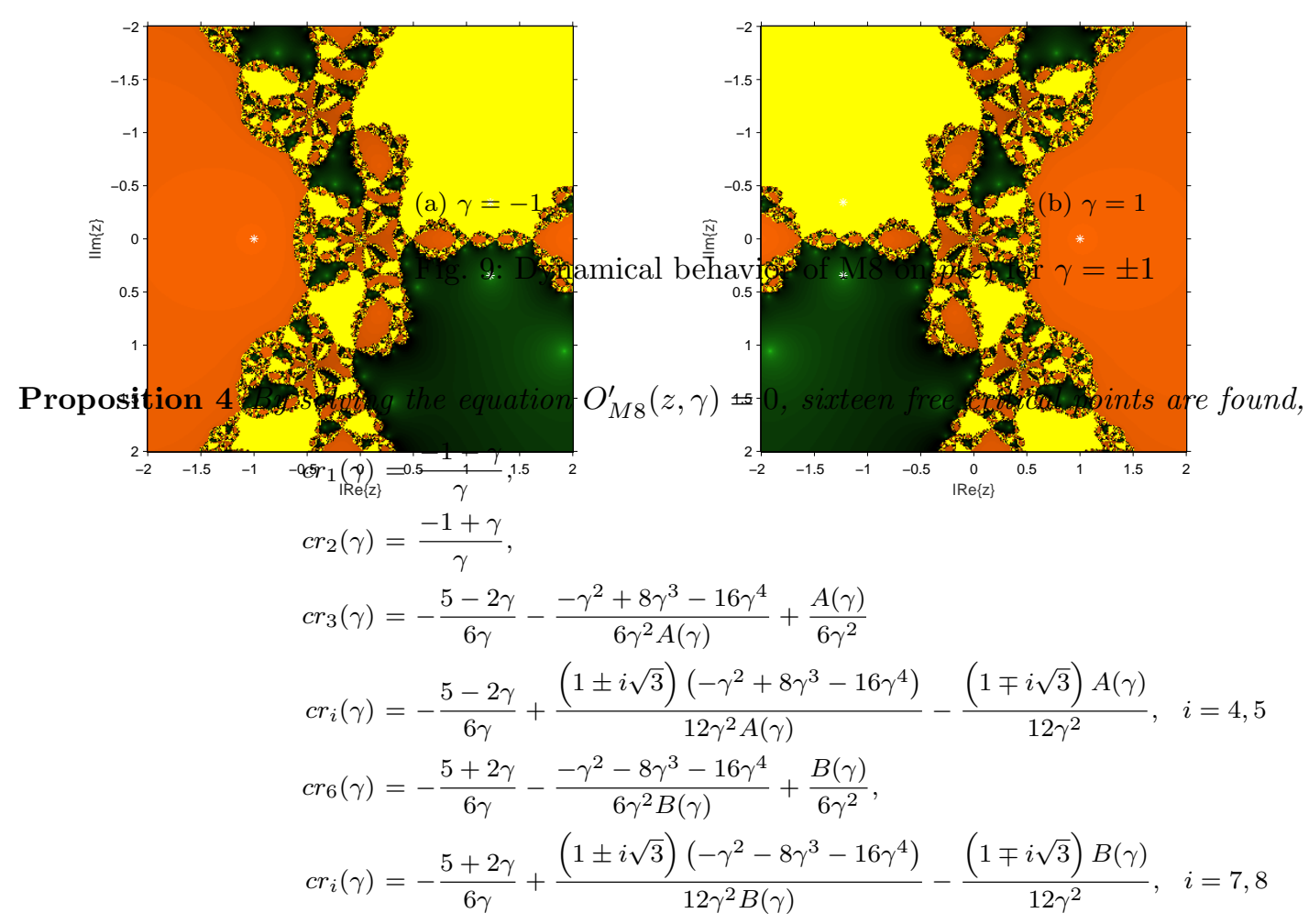

where

$$
A(\gamma)=\left(55 \gamma^{3}-12 \gamma^{4}+48 \gamma^{5}-64 \gamma^{6}+12 \sqrt{3} \sqrt{7 \gamma^{6}-3 \gamma^{7}+12 \gamma^{8}-16 \gamma^{9}}\right)^{1 / 3}
$$



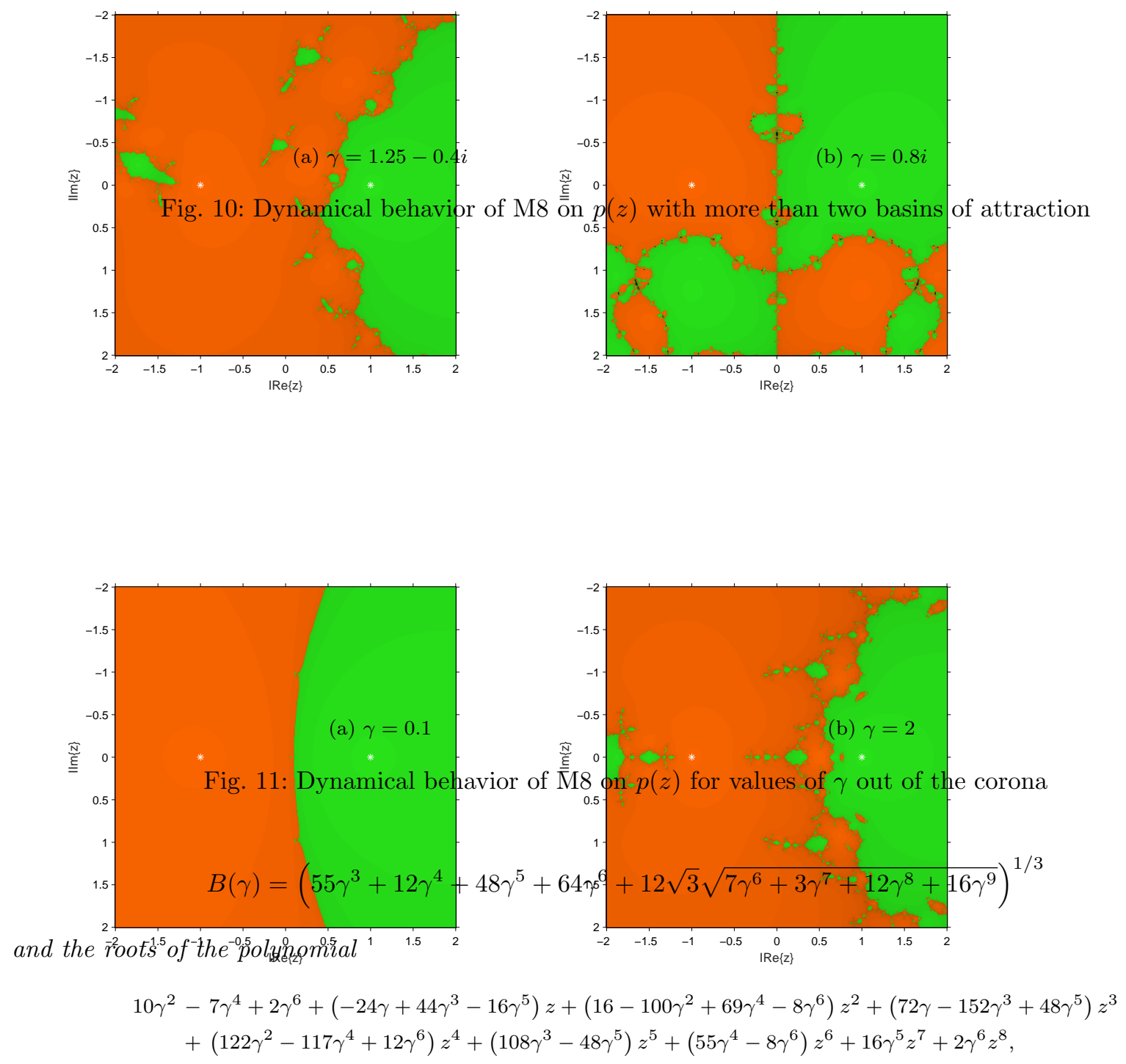

except in the following cases:

- If $\gamma=1$, then there exist eleven free critical points: $z=0, z=-2, z=1 / 2$,

$$
\begin{aligned}
& z=-\frac{7}{6}+\frac{1}{6}(179-12 \sqrt{114})^{1 / 3}+\frac{1}{6}(179+12 \sqrt{114})^{1 / 3} \\
& z=-\frac{7}{6}-\frac{1}{12}(1 \pm i \sqrt{3})(179-12 \sqrt{114})^{1 / 3}-\frac{1}{12}(1 \mp i \sqrt{3})(179+12 \sqrt{114})^{1 / 3}
\end{aligned}
$$

and the roots of $5-11 z-5 z^{2}+11 z^{3}+10 z^{4}+2 z^{5}$. 
- If $\gamma=-1$, then there are also eleven free critical points: $z=0, z=2, z=-1 / 2$,

$$
\begin{aligned}
& z=\frac{7}{6}-\frac{25}{6(179-12 \sqrt{114})^{1 / 3}}-\frac{1}{6}(179-12 \sqrt{114})^{1 / 3} \\
& z=\frac{7}{6}+\frac{25(1 \pm i \sqrt{3})}{12(179-12 \sqrt{114})^{1 / 3}}+\frac{1}{12}(1 \mp i \sqrt{3})(179-12 \sqrt{114})^{1 / 3}
\end{aligned}
$$

and the roots of $-5-11 z+5 z^{2}+11 z^{3}-10 z^{4}+2 z^{5}$.

- When $\gamma=\frac{1}{2}$, there exist fourteen free critical points, $z=-3$,

$$
\begin{aligned}
& z=-2+\frac{1}{3}(81-54 \sqrt{2})^{1 / 3}+(3+2 \sqrt{2})^{1 / 3}, \\
& z=-2-\frac{1}{2}(3+2 \sqrt{2})^{1 / 3}(1-i \sqrt{3})-\frac{1}{6}(81-54 \sqrt{2})^{1 / 3}(1+i \sqrt{3}), \\
& z=-2-\frac{1}{6}(81-54 \sqrt{2})^{1 / 3}(1-i \sqrt{3})-\frac{1}{2}(3+2 \sqrt{2})^{1 / 3}(1+i \sqrt{3}), \\
& z=\frac{1}{3}\left(-4+(53-6 \sqrt{78})^{1 / 3}+(53+6 \sqrt{78})^{1 / 3}\right), \\
& z=-\frac{4}{3}-\frac{1}{6}(1 \pm i \sqrt{3})(53-6 \sqrt{78})^{1 / 3}-\frac{1}{6}(1 \mp i \sqrt{3})(53+6 \sqrt{78})^{1 / 3}
\end{aligned}
$$

and the roots of $67-291 z+137 z^{2}+455 z^{3}+293 z^{4}+91 z^{5}+15 z^{6}+z^{7}$.

- When $\gamma=-\frac{1}{2}$, there also exist fourteen free critical points, $z=3$,

$$
\begin{aligned}
& z=2-\frac{1}{(3-2 \sqrt{2})^{1 / 3}}-(3-2 \sqrt{2})^{1 / 3} \\
& z=2+\frac{1}{2}(3-2 \sqrt{2})^{1 / 3}(1-i \sqrt{3})+\frac{1+i \sqrt{3}}{2(3-2 \sqrt{2})^{1 / 3}}, \\
& z=2+\frac{1-i \sqrt{3}}{2(3-2 \sqrt{2})^{1 / 3}}+\frac{1}{2}(3-2 \sqrt{2})^{1 / 3}(1+i \sqrt{3}), \\
& z=\frac{4}{3}-\frac{1}{3(53-6 \sqrt{78})^{1 / 3}}-\frac{1}{3}(53-6 \sqrt{78})^{1 / 3}, \\
& z=\frac{4}{3}+\frac{1 \pm i \sqrt{3}}{6(53-6 \sqrt{78})^{1 / 3}}+\frac{1}{6}(1 \mp i \sqrt{3})(53-6 \sqrt{78})^{1 / 3}
\end{aligned}
$$

and the roots of $-67-291 z-137 z^{2}+455 z^{3}-293 z^{4}+91 z^{5}-15 z^{6}+z^{7}$.

- If $\gamma=\frac{i}{2}$, then the free critical points are eleven: $z= \pm 1-2 i$,

$$
\begin{aligned}
& z=\frac{-2+3 i}{2} \pm \frac{\sqrt{-1-8 i}}{2} \\
& z=\frac{2+3 i}{2} \pm \frac{\sqrt{-1+8 i}}{2}
\end{aligned}
$$

and the roots of $-19 i+81 z+126 i z^{2}-50 z^{3}-11 i z^{4}+z^{5}$.

- If $\gamma=-\frac{i}{2}$, then the free critical points are eleven: $z= \pm 1+2 i$,

$$
\begin{aligned}
& z=\frac{2-3 i}{2} \pm \frac{\sqrt{-1-8 i}}{2} \\
& z=\frac{-2-3 i}{2} \pm \frac{\sqrt{-1+8 i}}{2}
\end{aligned}
$$

and the roots of $19 i+81 z-126 i z^{2}-50 z^{3}+11 i z^{4}+z^{5}$.

- For $\gamma=\frac{1}{8}(-1+3 i \sqrt{3})$, there exist thirteen free critical points: $z=-\frac{5}{7}+\frac{6}{7} \sqrt{3} i, z=\frac{9}{7}+\frac{6}{7} \sqrt{3} i$ and the roots of an eighth-degree polynomial with complex coefficients. 
- For $\gamma=\frac{1}{8}(-1-3 i \sqrt{3})$, there are thirteen free critical points: $z=-\frac{5}{7}-\frac{6}{7} \sqrt{3} i, z=\frac{9}{7}-\frac{6}{7} \sqrt{3} i$ and the roots of an eighth-degree polynomial with complex coefficients.

- For $\gamma=\frac{1}{8}(1+3 i \sqrt{3})$, there are thirteen free critical points: $z=\frac{5}{7}-\frac{6}{7} \sqrt{3} i, z=-\frac{9}{7}-\frac{6}{7} \sqrt{3} i$ and the roots of an eighth-degree polynomial with complex coefficients.

- For $\gamma=\frac{1}{8}(1-3 i \sqrt{3})$, there exist thirteen free critical points: $z=\frac{5}{7}+\frac{6}{7} \sqrt{3} i, z=-\frac{9}{7}+\frac{6}{7} \sqrt{3} i$ and the roots of an eighth-degree polynomial with complex coefficients.
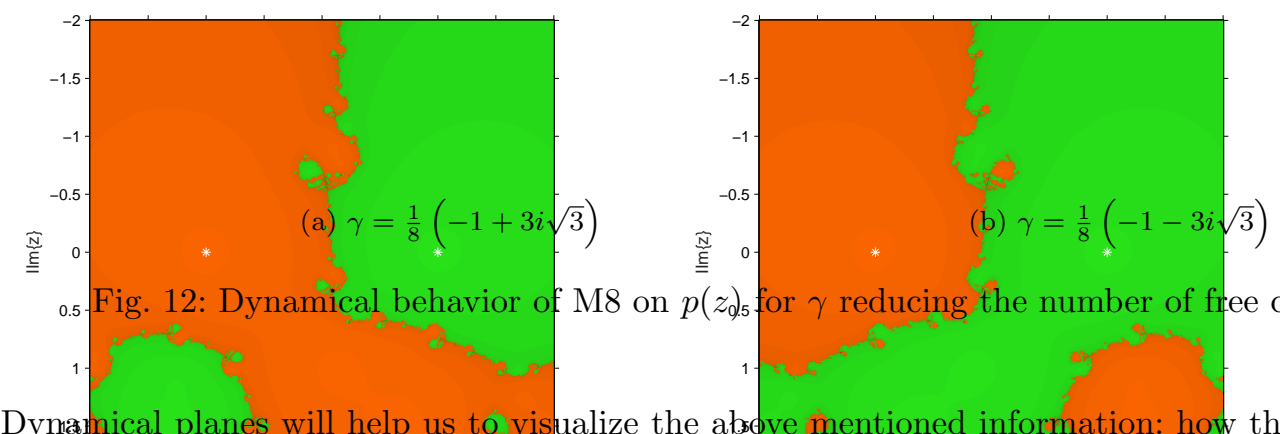

Dynamical planes will help us to visualize the above mentioned information: how the values of parameters that simplify the fixed point operator can yield to more stable behavior (see Figure 12) and how those values of

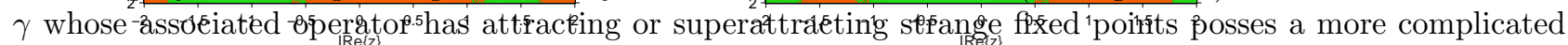
even dangerous numerical behavior.

\section{Design of new parametric methods with memory}

This section deals with developing methods with memory from the classes modified Steffensen, M4 and M8. By using $\gamma$ as accelerating parameter, we derive three methods with memory that increase the efficiency indices in such a way that no new functional evaluations are required regarding the original classes. The technique used to get this aim firstly appears in the text of Traub [10] and later was developed by Petković et al. (see [6]). Recent papers developing this kind of methods are [19,20,21] and the references therein.

Looking at the error equations of damped Steffensen (1), M4 (3) and M8 (5), it is clear that if $1+\gamma f^{\prime}(\alpha)=0$, then the corresponding order of convergence is improved. This can be done if $\gamma=\frac{-1}{f^{\prime}(\alpha)}$. But this is not possible, since $\alpha$ is not known. However, we use approximations for $f^{\prime}(\alpha)$ based on highest-degree interpolation polynomial by using available data, to increase efficiency indices as high as possible. In what follows, we describe this process in detail.

3.1 Method with memory from modified Steffensen's family

To construct a method with memory from damped Steffensen's class, considering its error equation (1), we should approximate $f^{\prime}(\alpha)$ with $N_{2}^{\prime}\left(x_{n}\right)$ where

$$
N_{2}(t)=N_{2}\left(t ; x_{n}, x_{n-1}, w_{n-1}\right)=f\left(x_{n-1}\right)+\left(t-x_{n-1}\right) f\left[x_{n-1}, w_{n-1}\right]+\left(t-x_{n-1}\right)\left(t-w_{n-1}\right) f\left[x_{n-1}, w_{n-1}, x_{n}\right],
$$


is Newton's interpolation polynomial of second degree. Let us suppose that $x_{0}$ and $\gamma_{0}$ are suitably chosen. Therefore, we propose the following method with memory

$$
\left\{\begin{array}{l}
\text { Initial step: } x_{0}, \gamma_{0} \text { are given, then } \\
w_{n}=x_{n}+\gamma_{n} f\left(x_{n}\right), \\
x_{n+1}=x_{n}-\frac{f\left(x_{n}\right)}{f\left[x_{n}, w_{n}\right]}, \\
\gamma_{n+1}=\frac{-1}{N_{2}^{\prime}\left(x_{n+1}\right)}, n=0,1,2, \ldots
\end{array}\right.
$$

The next result determines the order of convergence of the scheme (6), that we will denote by M3.

Theorem 1 Let $\alpha$ be a simple zero of a sufficiently differentiable function $f: D \subset \mathbb{R} \rightarrow \mathbb{R}$ in an open interval D. If $x_{0}$ is sufficiently close to $\alpha$, then the order of convergence of method with memory (6) is at least 3.

Proof Let $\left\{x_{n}\right\}$ be a convergent sequence to $\alpha$, generated by (6), with convergence order $r$. Symbolically

$$
e_{n+1} \sim e_{n}^{r}=e_{n-1}^{r^{2}},
$$

where $e_{n}=x_{n}-\alpha$. Let $e_{n, w}=w_{n}-\alpha$ be the error on step $w_{n}$. Also, let us suppose that the sequence $\left\{w_{n}\right\}$ has convergence order $p$, that is,

$$
e_{n, w} \sim e_{n}^{p}=\left(e_{n-1}^{r}\right)^{p}=e_{n-1}^{r p} .
$$

Since $f^{\prime}(\alpha) \approx N_{2}^{\prime}\left(x_{n}\right)$, then

$$
1+\gamma_{n} f^{\prime}(\alpha) \sim e_{n-1} e_{n-1, w}=e_{n-1} e_{n-1}^{p}=e_{n-1}^{1+p} .
$$

From (9) and the error equations of the sequences $\left\{x_{n}\right\}$ and $\left\{w_{n}\right\}$, we obtain

$$
e_{n, w} \sim\left(1+\gamma_{n} f^{\prime}(\alpha)\right) e_{n}=e_{n-1}^{1+p+r}
$$

and

$$
e_{n+1} \sim\left(1+\gamma_{n} f^{\prime}(\alpha)\right) e_{n}^{2}=e_{n-1}^{1+p+2 r} .
$$

Now, to obtain the convergence order $r$, it is enough to equate the powers of $e_{n-1}$ on the right-hand sides of the relations (8) and (10), and (7) and (11). As a result, we get

$$
\left\{\begin{array}{l}
r p-r-p=1 \\
r^{2}-2 r-p=1
\end{array}\right.
$$

The unique positive solution of this system is $r=3$ and $p=2$, which proves the theorem.

In Theorem 1 we have proved that the convergence order of the method with memory (6) is at least 3 by using the same functional evaluations as classical Steffensen's method. Therefore, its efficiency index is $3^{1 / 2} \approx 1.73$ which is higher than that of Steffensen's method of order two, $2^{1 / 2} \approx 1.41$.

\subsection{Method with memory from M4}

Now, we present a new method with memory from the two-point method without memory M4. Taking into account its error equation (3), to improve its order of convergence, we should approximate $f^{\prime}(\alpha)$ by using all the available functional evaluations in iteration $x_{n-1}$ and $x_{n}, N_{3}^{\prime}\left(x_{n}\right)$, where

$$
\begin{aligned}
N_{3}(t)= & N_{3}\left(t ; x_{n}, x_{n-1}, w_{n-1}, y_{n-1}\right) \\
= & f\left(x_{n-1}\right)+\left(t-x_{n-1}\right) f\left[x_{n-1}, w_{n-1}\right]+\left(t-x_{n-1}\right)\left(t-w_{n-1}\right) f\left[x_{n-1}, w_{n-1}, x_{n}\right] \\
& +\left(t-x_{n-1}\right)\left(t-w_{n-1}\right)\left(t-y_{n-1}\right) f\left[x_{n-1}, w_{n-1}, y_{n-1}, x_{n}\right]
\end{aligned}
$$


is Newton's interpolation polynomial of third degree. Let us assume that $x_{0}$ and $\gamma_{0}$ are suitably chosen. Therefore, we propose the following method with memory

$$
\left\{\begin{array}{l}
\text { Initial step: } x_{0}, \gamma_{0} \text { are given, then } \\
w_{n}=x_{n}+\gamma_{n} f\left(x_{n}\right) \\
y_{n}=x_{n}-\gamma_{n} \frac{f\left(x_{n}\right)^{2}}{f\left(w_{n}\right)-f\left(x_{n}\right)}, \\
x_{n+1}=y_{n}-\frac{w_{n}-y_{n}}{\left(x_{n}-y_{n}\right) \gamma_{n}}-\frac{\left(x_{n}-y_{n}\right)}{\left(w_{n}-y_{n}\right) \gamma_{n} f\left(x_{n}\right)} f\left(w_{n}\right)-\frac{w_{n}+x_{n}-2 y_{n}}{\left(x_{n}-y_{n}\right)\left(w_{n}-y_{n}\right)} f\left(y_{n}\right) \\
\gamma_{n+1}=\frac{-1}{N_{3}^{\prime}\left(x_{n+1}\right)}, n=0,1,2, \ldots
\end{array}\right.
$$

The next result determines the convergence order of this method with memory, that will be denoted by M6.

Theorem 2 Let $\alpha$ be a simple zero of a sufficiently differentiable function $f: D \subset \mathbb{R} \rightarrow \mathbb{R}$ in an open interval D. If $x_{0}$ is sufficiently close to $\alpha$, then the order of convergence of method with memory (13) is, at least, 6 .

Proof Let $\left\{x_{n}\right\}$ be a convergent sequence to $\alpha$, generated by (13), with order of convergence $r$. Symbolically

$$
e_{n+1} \sim e_{n}^{r}=e_{n-1}^{r^{2}}
$$

where $e_{n}=x_{n}-\alpha$. Let $e_{n, w}=w_{n}-\alpha$ and $e_{n, y}=y_{n}-\alpha$ be the error equations of $w_{n}$ and $y_{n}$. Also, let us assume that the sequences $\left\{w_{n}\right\}$ and $\left\{y_{n}\right\}$ have order of convergence $p$ and $q$, respectively, that is,

$$
e_{n, w} \sim e_{n}^{p}=\left(e_{n-1}^{r}\right)^{p}=e_{n-1}^{r p}
$$

and

$$
e_{n, y} \sim e_{n}^{q}=\left(e_{n-1}^{r}\right)^{q}=e_{n-1}^{r q}
$$

Since $f^{\prime}(\alpha) \approx N_{3}^{\prime}\left(x_{n}\right)$, then

$$
1+\gamma_{n} f^{\prime}(\alpha) \sim e_{n-1} e_{n-1, w} e_{n-1, y}=e_{n-1} e_{n-1}^{p} e_{n-1}^{q}=e_{n-1}^{1+p+q} .
$$

From (17) and the error equations of the sequences $\left\{x_{n}\right\},\left\{w_{n}\right\}$, and $\left\{y_{n}\right\}$, we obtain

$$
\begin{aligned}
& e_{n, w} \sim\left(1+\gamma_{n} f^{\prime}(\alpha)\right) e_{n}=e_{n-1}^{1+p+q+r} \\
& e_{n, y} \sim\left(1+\gamma_{n} f^{\prime}(\alpha)\right) e_{n}^{2}=e_{n-1}^{1+p+q+2 r}
\end{aligned}
$$

and

$$
e_{n+1} \sim\left(1+\gamma_{n} f^{\prime}(\alpha)\right)^{2} e_{n}^{4}=e_{n-1}^{2+2 p+2 q+4 r}
$$

Now, to make equal the powers of $e_{n-1}$ on the right-hand sides of the relations (15) and (18), (16) and (19), and (14) and (20) we get the system

$$
\left\{\begin{array}{l}
r p-r-p-q=1 \\
r q-2 r-p-q=1 \\
r^{2}-4 r-2 p-2 q=2
\end{array}\right.
$$

The unique positive solution of this system is $r=6, q=3$, and $p=2$ and this finishes the proof.

In Theorem 2, we have proved that the convergence order of the method with memory (13) is at least 6 by using the same functional evaluations as its partner without memory (2). Therefore, its efficiency index is $6^{1 / 3} \approx 1.81$ which is higher than that of $\mathrm{M} 4,4^{1 / 3} \approx 1.58$. 
3.3 Method with memory from M8

We here deduce a novel method with memory from the three-point family without memory M8, by using the same technique as in the previous subsections. In this case, we should approximate $f^{\prime}(\alpha)$ with $N_{4}^{\prime}\left(x_{n}\right)$ where

$$
\begin{aligned}
N_{4}(t)= & N_{4}\left(t ; x_{n}, x_{n-1}, w_{n-1}, y_{n-1}, z_{n-1}\right) \\
= & f\left(x_{n-1}\right)+\left(t-x_{n-1}\right) f\left[x_{n-1}, w_{n-1}\right]+\left(t-x_{n-1}\right)\left(t-w_{n-1}\right) f\left[x_{n-1}, w_{n-1}, x_{n}\right] \\
& \quad+\left(t-x_{n-1}\right)\left(t-w_{n-1}\right)\left(t-y_{n-1}\right) f\left[x_{n-1}, w_{n-1}, y_{n-1}, x_{n}\right] \\
& \quad+\left(t-x_{n-1}\right)\left(t-w_{n-1}\right)\left(t-y_{n-1}\right)\left(t-z_{n-1}\right) f\left[x_{n-1}, w_{n-1}, y_{n-1}, z_{n-1}, x_{n}\right]
\end{aligned}
$$

is Newton's interpolation polynomial of fourth degree. Let us suppose that $x_{0}$ and $\gamma_{0}$ are suitably chosen. Therefore, we propose the following scheme with memory

$$
\left\{\begin{array}{l}
\text { Initial step: } x_{0}, \gamma_{0} \text { are given, then } \\
w_{n}=x_{n}+\gamma_{n} f\left(x_{n}\right) \\
y_{n}=x_{n}-\gamma_{n} \frac{f\left(x_{n}\right)^{2}}{f\left(w_{n}\right)-f\left(x_{n}\right)}, \quad f\left(y_{n}\right) \\
z_{n}=y_{n}-\frac{w_{n}-y_{n}}{\left(x_{n}-y_{n}\right) \gamma_{n}}-\frac{\left(x_{n}-y_{n}\right)}{\left(w_{n}-y_{n}\right) \gamma_{n} f\left(x_{n}\right)} f\left(w_{n}\right)-\frac{w_{n}+x_{n}-2 y_{n}}{\left(x_{n}-y_{n}\right)\left(w_{n}-y_{n}\right)} f\left(y_{n}\right) \\
x_{n+1}=z_{n}-\frac{f z_{1}+H_{2}+H_{3}+H_{4}}{H_{1}},
\end{array}\right.
$$

where $H_{i}, i=1,2,3,4$ are defined as before.

In the next result, we determine the order of convergence of this method, that we will denote by M12.

Theorem 3 Let $\alpha$ be a simple zero of a sufficiently differentiable function $f: D \subset \mathbb{R} \rightarrow \mathbb{R}$ in an open interval D. If $x_{0}$ is sufficiently close to $\alpha$, then the order of convergence of method with memory (21) is, at least, 12.

Proof Let $\left\{x_{n}\right\}$ be a convergent sequence to $\alpha$, generated by (21) with convergence order $r$. Symbolically

$$
e_{n+1} \sim e_{n}^{r}=e_{n-1}^{r^{2}},
$$

where $e_{n}=x_{n}-\alpha$. Let $e_{n, w}=w_{n}-\alpha, e_{n, y}=y_{n}-\alpha$, and $e_{n, z}=z_{n}-\alpha$ be the error equations of the different steps. Also, suppose that the sequences $\left\{w_{n}\right\},\left\{y_{n}\right\}$, and $\left\{z_{n}\right\}$ have convergence orders $p, q$, and $v$, respectively, that is,

$$
\begin{aligned}
& e_{n, w} \sim e_{n}^{p}=\left(e_{n-1}^{r}\right)^{p}=e_{n-1}^{r p}, \\
& e_{n, y} \sim e_{n}^{q}=\left(e_{n-1}^{r}\right)^{q}=e_{n-1}^{r q},
\end{aligned}
$$

and

$$
e_{n, z} \sim e_{n}^{v}=\left(e_{n-1}^{r}\right)^{v}=e_{n-1}^{r v}
$$

Since $f^{\prime}(\alpha) \approx N_{4}^{\prime}\left(x_{n}\right)$, then

$$
1+\gamma_{n} f^{\prime}(\alpha) \sim e_{n-1} e_{n-1, w} e_{n-1, y} e_{n-1, z}=e_{n-1} e_{n-1}^{p} e_{n-1}^{q} e_{n-1}^{v}=e_{n-1}^{1+p+q+v} .
$$

By using (26) and the error equations of sequences $\left\{x_{n}\right\},\left\{w_{n}\right\},\left\{y_{n}\right\}$, and $\left\{z_{n}\right\}$, we obtain

$$
\begin{gathered}
e_{n, w} \sim\left(1+\gamma_{n} f^{\prime}(\alpha)\right) e_{n}=e_{n-1}^{1+p+q+v+r}, \\
e_{n, y} \sim\left(1+\gamma_{n} f^{\prime}(\alpha)\right) e_{n}^{2}=e_{n-1}^{1+p+q+v+2 r}, \\
e_{n, z} \sim\left(1+\gamma_{n} f^{\prime}(\alpha)\right)^{2} e_{n}^{4}=e_{n-1}^{2+2 p+2 q+2 v+4 r}
\end{gathered}
$$

and

$$
e_{n+1} \sim\left(1+\gamma_{n} f^{\prime}(\alpha)\right)^{4} e_{n}^{8}=e_{n-1}^{4+4 p+4 q+4 v+8 r}
$$


Now, to obtain the convergence order $r$, it is enough to equate the powers of $e_{n-1}$ on the right-hand sides of the relations (23) and (27), (24) and (28), (25) and (29), and (22) and (30). Then, we obtain the system

$$
\left\{\begin{array}{l}
r p-r-p-q-v=1 \\
r q-2 r-p-q-v=1 \\
r v-4 r-2 p-2 q-2 v=2 \\
r^{2}-8 r-4 p-4 q-4 v=4
\end{array}\right.
$$

The unique positive solution of this system is $r=12, v=6, q=3$, and $p=2$, which proves the theorem.

In Theorem 3, we have proved that the convergence order of the method with memory (21) is at least 12 , by using the same number of functional evaluations as the original method without memory M8. Therefore, its efficiency index is $12^{1 / 4} \approx 1.86$, in contrast with that of order four, $8^{1 / 4} \approx 1.68$.

Remark 4 The proposed methods with memory M3, M6 and M12 can be extended in a straightforward way to the multidimensional case, $F(x)=0$. However, the existence of evaluations of function $F$ in the denominator of the iterative expression make it necessary to use the divided difference operator $[x, y ; F]$ (see, for instance [22, 23]), which yields to a higher computational cost.

\section{Comparing the dynamics of the proposed methods}

In this section we will compare, under a dynamical point of view, the behavior of the proposed methods M3, M6 and M12 with their respective partners without memory modified Steffensen, M4 and M8. To get this aim, we will check the dynamical behavior of these methods with memory by using as starting values of the accelerating parameter $\gamma_{0}$ those values of parameter $\gamma$ that have provided in Section 2 specially stable or unstable performances. We will use the algorithms designed in [18] and the same number of points and iterations than in previous sections.

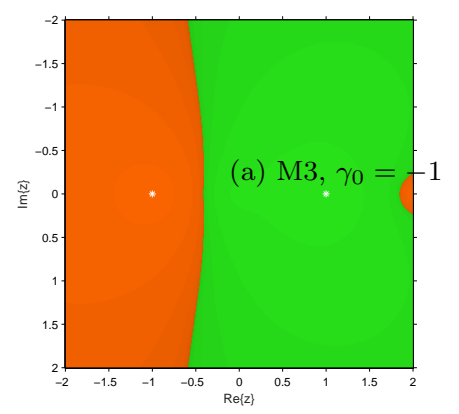

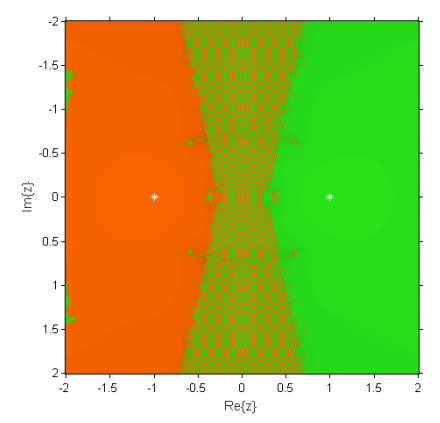

(b) M6, $\gamma_{0}=-1$
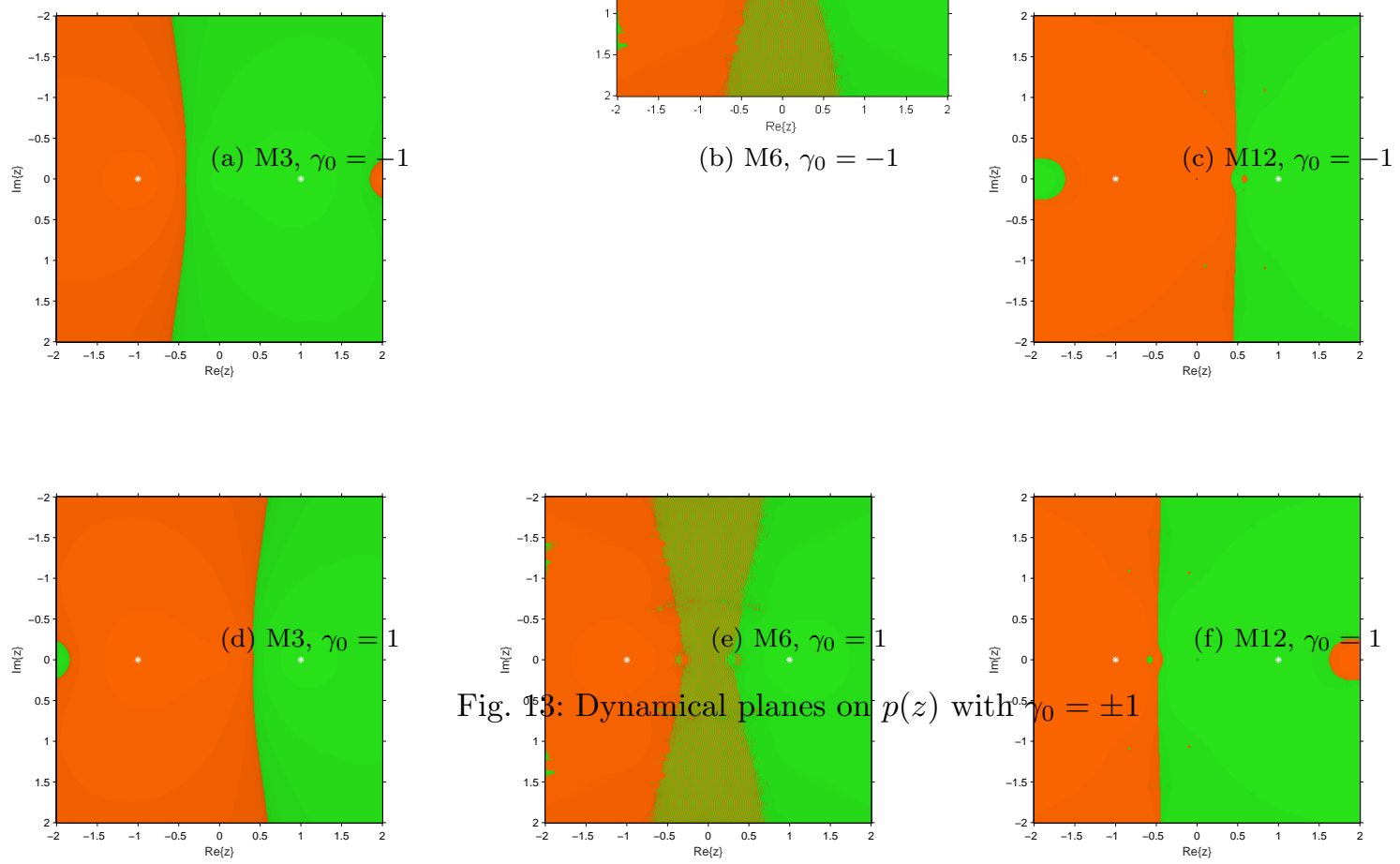
Let us remember that one of the special features of M4 and M8 on the quadratic polynomial $p(z)$ was the fact of loosing one of the roots as a fixed point when $\gamma= \pm 1$, and even its replacement by two attractive strange fixed points. Now, we apply M3, M6 and M12 on $p(z)$ by using these values of the parameter as starting ones, and correcting them in the successive steps of the process by the appropriate expressions of each method. Then, we check that the use of the memory in the iterative procedures, not only widen the areas of convergence of all the roots, but also hold the roots as fixed points of the associated operator. Moreover, no attracting fixed points appear in Figure 13. The central region of dynamical planes in Figures 13b and 13e correspond to small mixed preimages of the basin of convergence of the roots $z=-1$ and $z=1$.
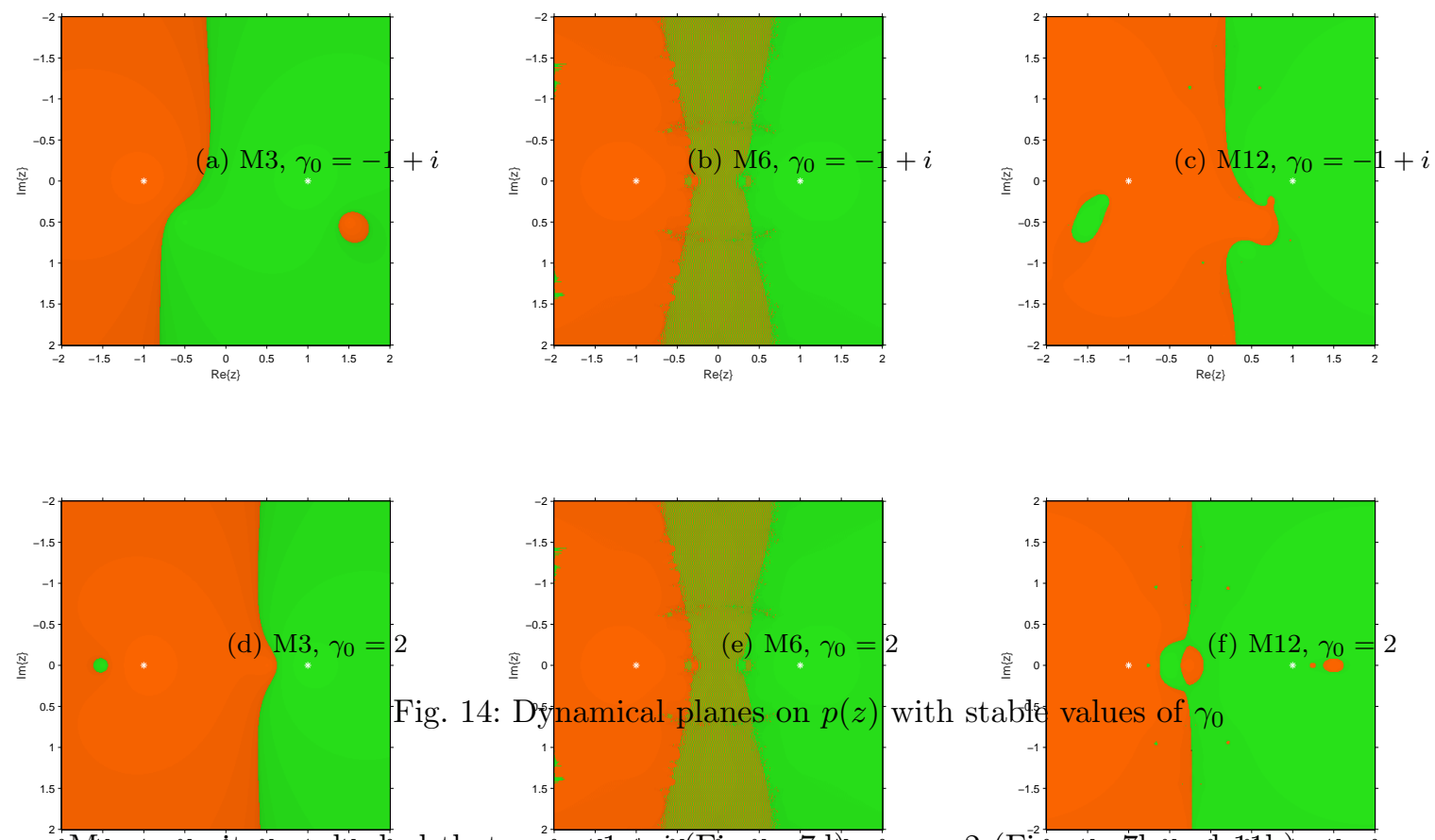

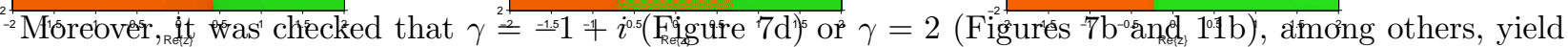
stable behavior of M4 and M8, but with black regions of no convergence in same cases. When methods with memory are applied on $p(z)$ by using the same values of parameter (see Figure 14), it is observed that the basins of convergence are wider and Julia set is simpler, except in case of M6.

Let us remember that there were values of $\gamma$ that yield to very dangerous behavior of the fixed point operator in M4 and M8: different basins of attracting strange fixed points appeared and also regions black regions of no convergence (see Figures 4,6 or 10). Now, we present the behavior of some of their with-memory partners, noting that there exist only convergence to the roots of $p(z)$ (see Figure 15).

Finally, let us consider now a cubic polynomial, $q(z)=z^{3}+4 z^{2}-10$. It has three roots, $\alpha_{1}=1.36523$, $\alpha_{2}=-2.682615+0.358259 i$ and $\alpha_{3}=-2.682615-0.358259 i$, so in Figure 16 four different colors will be used: orange, clear blue and green for the convergence to the roots, and black for no convergence. The improvement of the regions of good starting points is great, mostly for modified Steffensen' and M4 methods and their corresponding schemes with memory. The considered value of the parameter is $\gamma=1$ in all cases (in the methods with memory, it has been the initial value of $\gamma$ ). The improvement of the regions of good starting points is great, mostly for modified Steffensen' and M4 methods and their corresponding schemes with memory, but it can be observed that the structure of the Julia set is simpler when the order of the method with memory increases from 6 to 12 . 


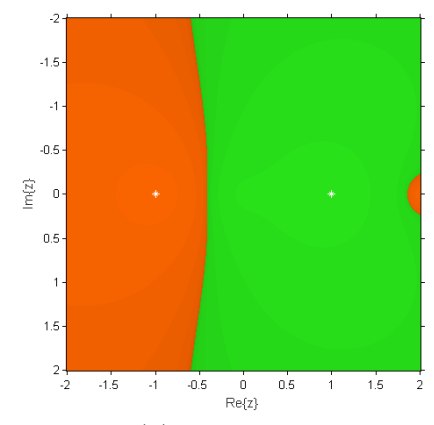

(a) $\mathrm{M} 3, \gamma_{0}=-0.98$
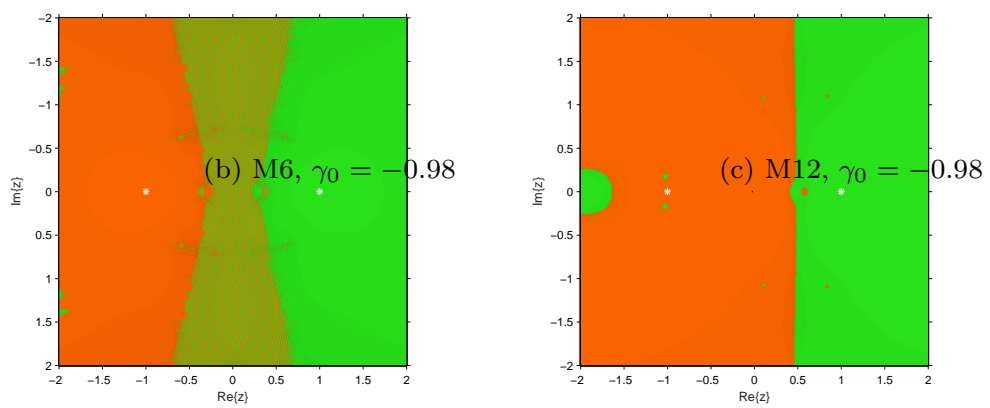

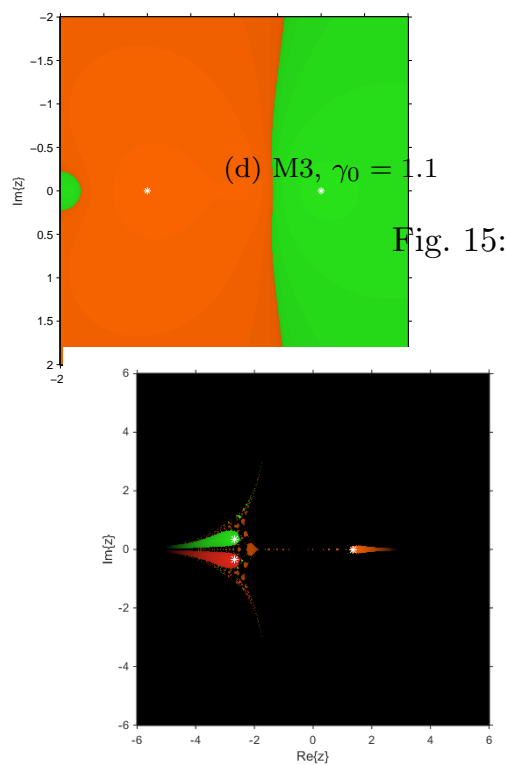

(a) Damped Steffensen, $\gamma_{0}=$
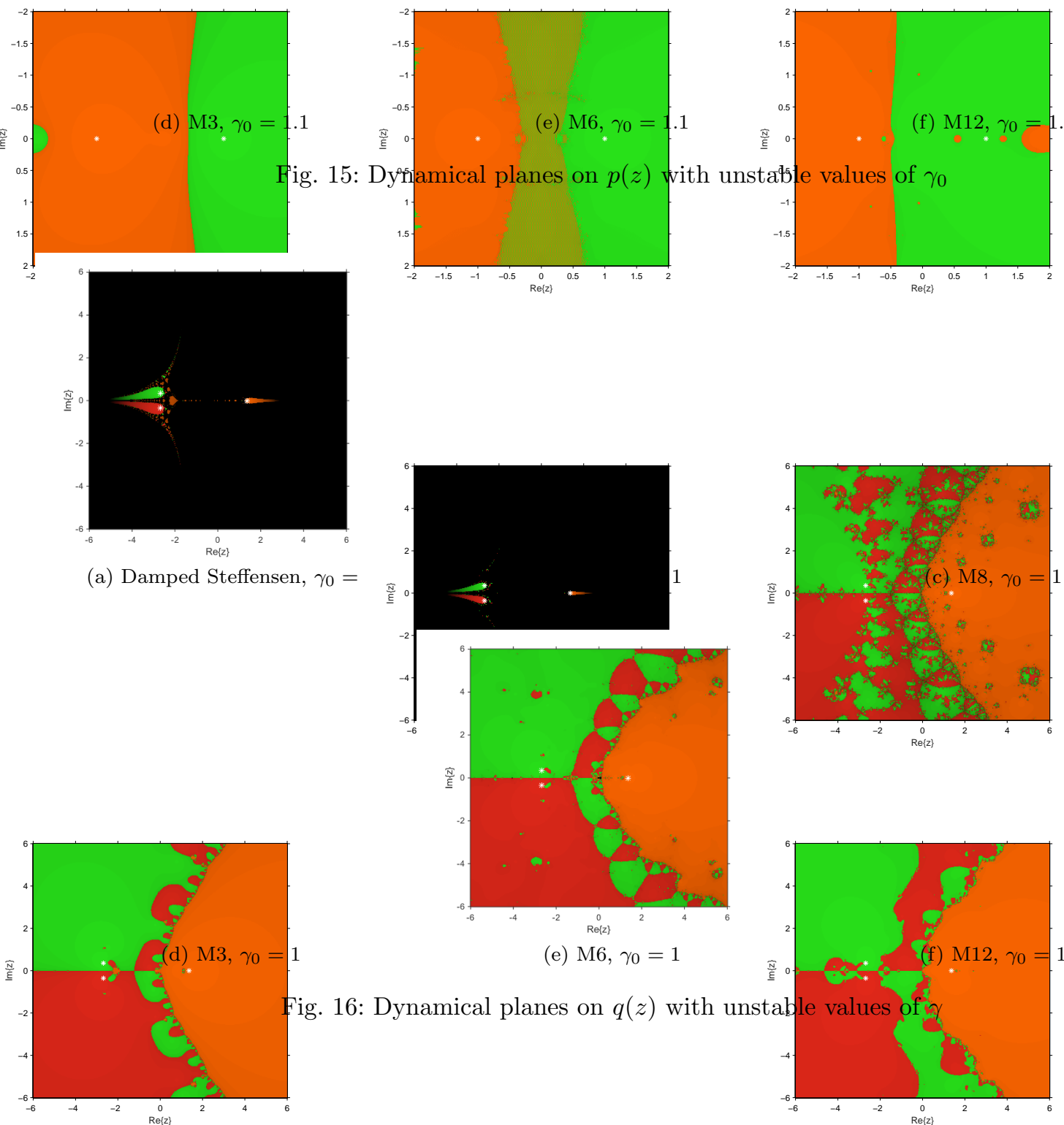

(e) $\mathrm{M} 6, \gamma_{0}=1$
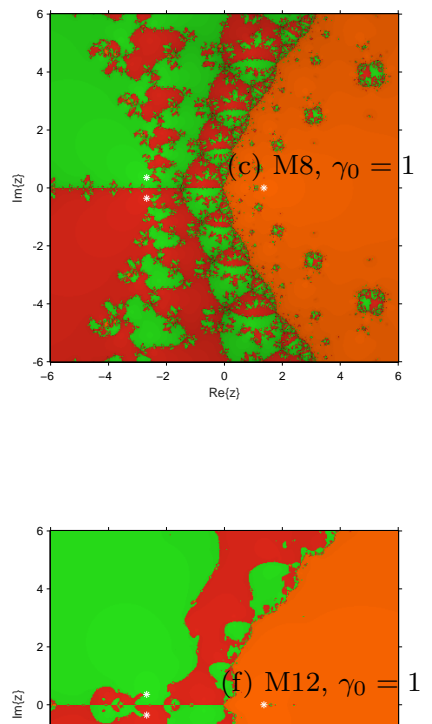

ig. 16: Dynamical planes on $q(z)$ with unstable values of $y$

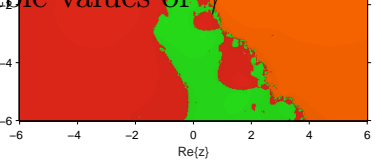




\section{Numerical performances and comparisons}

In the following, we compare the proposed methods with memory with their optimal without memory partners to estimate the solution of the physical problem of calculating the friction factor in a pipe, and also on some academic examples. In general, $b(-a)$ stands for $b \times 10^{-a}$ and the theoretical order of convergence of each procedure will be compared with the computational order of convergence (COC), defined in [24] as

$$
\mathrm{COC}=\frac{\ln \left(\left|x_{n+1}-\alpha\right| /\left|x_{n}-\alpha\right|\right)}{\ln \left(\left|x_{n}-\alpha\right| /\left|x_{n-1}-\alpha\right|\right)} .
$$

\subsection{Performance on the calculation of the friction factor of a pipe}

To check the numerical behavior of these methods, an applied chemical problem is analyzed: when the flow within a round-section pipe is analyzed, different models are used (see, for example, [25]); these models show experimental relationship among the different variables in the flow transport in a pipe, such as Reynolds number $R e$ with the longitude, inner diameter and rugosity of the pipe $\varepsilon_{r}$ and its friction factor $f_{f}$.

Colebrook-White equation is one of the more precise and wide-rank ways to calculate the friction factor $f_{f}$ associated to a pipe, but is an implicit function that must be solved in an iterative way,

$$
\frac{1}{\sqrt{f_{f}}}=-2.0 \log _{10}\left(\frac{\varepsilon_{r}}{3.7065}+\frac{2.5226}{R e \sqrt{f_{f}}}\right) .
$$

In the following, a particular case is shown corresponding to the values of Reynolds number $R e=4 \cdot 10^{3}$ and rugosity factor $\varepsilon_{r}=1 \cdot 10^{-4}$ (in this case, the friction factor is $f_{f} \approx 0.0401$ ). In any method with memory, the second iterate is calculated by using $\gamma_{0}=-0.01$. The behavior of Newton's method, modified Steffensen (M2) and also the proposed schemes with memory as well as their without memory partners is shown on this

\begin{tabular}{|c|c|c|c|c|c|}
\hline Method & $x_{0}$ & iter & $\left|x_{k+1}-x_{k}\right|$ & $\left|f\left(x_{k+1}\right)\right|$ & e-time (sec) \\
\hline \multirow{4}{*}{ NM } & 0.03 & 4 & $7.6372(-8)$ & $7.5921(-12)$ & 0.2734 \\
\hline & 0.05 & 4 & $4.5254(-8)$ & $2.6657(-12)$ & 0.2391 \\
\hline & 0.1 & nc & - & - & - \\
\hline & 0.12 & $\mathrm{nc}$ & - & - & - \\
\hline \multirow{4}{*}{ M2 } & 0.03 & nc & - & - & - \\
\hline & 0.05 & nc & - & - & - \\
\hline & 0.1 & $\mathrm{nc}$ & - & - & - \\
\hline & 0.12 & $\mathrm{nc}$ & - & - & - \\
\hline \multirow{4}{*}{ M3 } & 0.03 & 2 & $3.5904(-6)$ & $\begin{array}{c}1.6165(-13) \\
\end{array}$ & 0.0437 \\
\hline & 0.05 & 2 & $7.3713(-7)$ & $8.8818(-16)$ & 0.0391 \\
\hline & 0.1 & 4 & $8.7761(-10)$ & 0 & 0.0516 \\
\hline & 0.12 & 4 & $9.6096(-7)$ & $2.7197(-15)$ & 0.0437 \\
\hline \multirow{4}{*}{ M4 } & 0.03 & nc & - & - & - \\
\hline & 0.05 & nc & - & - & - \\
\hline & 0.1 & nc & - & - & - \\
\hline & 0.12 & nc & - & - & - \\
\hline \multirow{4}{*}{ M6 } & 0.03 & 1 & $3.6651(-5)$ & $7.76698(-12)$ & 0.0453 \\
\hline & 0.05 & 1 & $0.0034(-1)$ & $7.1552(-9)$ & 0.0391 \\
\hline & 0.1 & 2 & 0.00015 & $1.5593(-9)$ & 0.0391 \\
\hline & 0.12 & 5 & $7.9806(-7)$ & $7.3754(-19)$ & 0.0547 \\
\hline \multirow{4}{*}{ M8 } & 0.03 & nc & - & - & - \\
\hline & 0.05 & 5 & $6.1324(-8)$ & $4.4409(-15)$ & 0.0469 \\
\hline & 0.1 & $\mathrm{nc}$ & - & - & - \\
\hline & 0.12 & $\mathrm{nc}$ & - & - & - \\
\hline \multirow{4}{*}{ M12 } & $\overline{0.03}$ & $\overline{1}$ & $2.4529(-8)$ & $\overline{0}$ & 0.0406 \\
\hline & 0.05 & 1 & $2.0693(-6)$ & 0 & 0.0422 \\
\hline & 0.1 & 3 & $2.8904(-8)$ & $9.9298(-26)$ & 0.0422 \\
\hline & 0.12 & 4 & $1.6971(-8)$ & $3.8401(-19)$ & 0.0594 \\
\hline
\end{tabular}
problem, by using $\gamma=\gamma_{0}=-0.01$.

Table 1: Performance of the methods for $R e=4 \cdot 10^{3}$ and $\varepsilon_{r}=1 \cdot 10^{-4}$ 
(a) $\mathrm{M} 2, \gamma_{0}=-0.01$
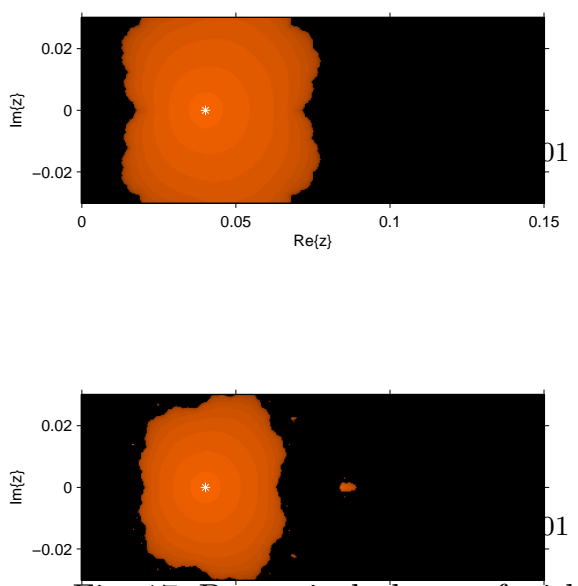

Fig. 17: Dyønamical planes of with and without memory schemes on Colebrook-White equation (b) $\mathrm{M} 3, \gamma_{0}=-0.01$

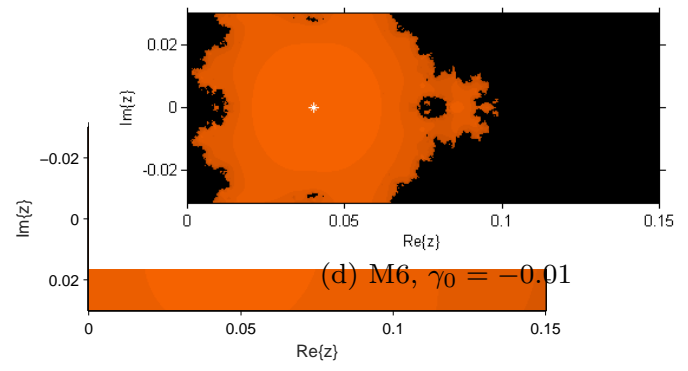

(f) $\mathrm{M} 12, \gamma_{0}=-0.01$

\begin{abstract}
Thoo estimatic meatns of and $\mid f_{0} .62 t$ oping criter
$x_{0}$ in all the
e following 1
) ( being $f$ oping criter
$x_{0}$ in all the a
e following i
) (being $f$ en $\left|x_{k+1}-x_{k}^{0.02}\right|$ ls. We show in II r of iterations inear equation tol or $\left|f\left(x_{k+1}\right)\right|<t$ le 1 the performance of er, the error estimatio hose root is the frictio have used different initial ew and known methods by last iteration, $\left|x_{k+1}-x_{k}\right|$ or) and the mean elapsed

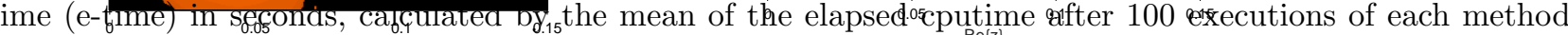
(by using a processor linţel(R) Core (TM) i5-3210M CPU @ 2.50 GHz (64-bit Machine), OS X V.10.9.4, RAM Memory 16 GB, $1600 \mathrm{MHz}$ DDR3). Double precision arithmetics with 32 digits has been used, and the tolerance used in the stopping criterium has been tol $=10^{-8}$. When a method does not converge, it is marked in the table with 'nc'.
\end{abstract}

In Table 1 the computational order of convergence is not included, as it cannot be calculated in some cases (there is not enough iterations) and, when it is possible, the number of iterations is not significative to have an stable value. On the other hand, it can be observed that the performance of the proposed methods with memory improve the results obtained by Newton's method, not only in terms of number of iterations, but also in terms of convergence to the solution (when the initial estimation is not very close to the solution and Newton and the rest of optimal methods without memory M2, M4 and M8 fail) but overall in terms of execution time, being significatively lower in cases M3, M6 and M12. These results also tell us that in case of this physical problem, the use of method M3 is good enough to achieve the estimated solution with the required precision in a minimum elapsed time. Other problems may require higher order schemes with memory and their wider basins of convergence (respect their original schemes without memory) could be very useful.

In Figure 17 we have shown that the basin of attraction of each iterative method with memory improves the corresponding one of its partner without memory. Indeed, it is observed in Figure 17b that the best scheme for solving this specific problem is method M3. 


\subsection{Some academic examples}

In this section, we test our proposed methods with memory M6 and M12 and compare their results with some other methods with memory of the same order of convergence obtained by applying the same idea to other known methods. In this case, we have used variable precision arithmetics and we show the exact error $\left|x_{n}-\alpha\right|$ to approximate the zero $\alpha$ at the $n$ th-iteration (we will take $n=1,2,3$ ). We will use some test functions:

$$
\begin{aligned}
& f_{1}(x)=x^{3}+4 x^{2}-10, \alpha_{1}=1.36523, \text { and } \alpha_{2,3}=-2.682615 \pm 0.358259 i \\
& f_{2}(x)=x^{4}+\sin \left(\pi / x^{2}\right)-5, \alpha_{1}=\sqrt{2} \text { and } \alpha_{2}=-\sqrt{2}, \\
& f_{3}(x)=e^{-x^{2}+x+2}-1, \alpha_{1}=2 \text { and } \alpha_{2}=-1,
\end{aligned}
$$

In the following, we are going to introduce different methods with memory for the comparison, distinguishing between two- and three-point methods.

Kung and Traub in [26] proposed the following two-point optimal method without memory

$$
\left\{\begin{array}{l}
y_{n}=x_{n}-\frac{f\left(x_{n}\right)}{f\left[w_{n}, x_{n}\right]}, \\
x_{n+1}=y_{n}-\frac{f\left(y_{n}\right) f\left(w_{n}\right)}{\left(f\left(w_{n}\right)-f\left(y_{n}\right)\right) f\left[x_{n}, y_{n}\right]}, \quad n=0,1, \ldots
\end{array}\right.
$$

where $w_{n}=x_{n}+\gamma_{n} f\left(x_{n}\right)$. Taking into account the error equation of this method and following the procedure established in Section 3, we can construct the following scheme with memory of order six, which is denoted by KT6.

$$
\left\{\begin{array}{l}
x_{0}, w_{0}, \gamma_{0} \text { are given suitably, } \\
w_{n}=x_{n}+\gamma_{n} f\left(x_{n}\right) \\
y_{n}=x_{n}-\frac{f\left(x_{n}\right)}{f\left[w_{n}, x_{n}\right]} \\
x_{n+1}=y_{n}-\frac{f\left(y_{n}\right) f\left(w_{n}\right)}{\left(f\left(w_{n}\right)-f\left(y_{n}\right)\right) f\left[x_{n}, y_{n}\right]} \\
\gamma_{n+1}=-1 / N_{3}^{\prime}\left(x_{n+1}\right), \quad n=0,1, \ldots
\end{array}\right.
$$

The same idea applied to the optimal fourth-order method designed by Zheng et al. in [27] allows us to get the sixth-order scheme with memory described by the following iterative expression, that will be denoted by ZLH6.

$$
\left\{\begin{array}{l}
x_{0}, w_{0}, \gamma_{0} \text { are given suitably, } \\
w_{n}=x_{n}+\gamma_{n} f\left(x_{n}\right), \\
y_{n}=x_{n}-\frac{f\left(x_{n}\right)}{f\left[x_{n}, w_{n}\right]}, \quad f\left(y_{n}\right) \\
x_{n+1}=y_{n}-\frac{f\left[y_{n}, w_{n}\right]+f\left[y_{n}, x_{n}, w_{n}\right]\left(y_{n}-x_{n}\right)}{f}, \\
\gamma_{n+1}=-1 / N_{3}^{\prime}\left(x_{n+1}\right), \quad n=0,1, \ldots
\end{array}\right.
$$

Finally, the following iterative expression correspond to the sixth-order method obtained when the same procedure is applied on the optimal two-point scheme from Soleymani et al. in [28]. It will be denoted by S6.

$$
\left\{\begin{array}{l}
x_{0}, w_{0} \gamma_{0} \text { are given suitably, } \\
w_{n}=x_{n}+\gamma_{n} f\left(x_{n}\right) \\
y_{n}=x_{n}-\frac{f\left(x_{n}\right)}{f\left[w_{n}, x_{n}\right]}, \\
x_{n+1}=y_{n}-\frac{f\left(y_{n}\right)}{f\left[y_{n}, x_{n}\right]+f\left[w_{n}, x_{n}, y_{n}\right]\left(y_{n}-x_{n}\right)+\left(y_{n}-x_{n}\right)\left(y_{n}-w_{n}\right)} \\
\gamma_{n+1}=-1 / N_{3}^{\prime}\left(x_{n+1}\right), \quad n=0,1,2, \ldots
\end{array}\right.
$$

Tables 2, 3 and 4 show numerical results for two-point method with memory mentioned above. It is clear that all this method behave very well practically and confirm their theoretical results.

In an analogous way as it was made in the previous subsection, an iterative scheme with memory of order of convergence twelve can be obtained from three-point procedure by Kung and Traub [26], by using the technique 


\begin{tabular}{|l|c|c|c|c|}
\hline Methods & $\left|x_{1}-\alpha\right|$ & $\left|x_{2}-\alpha\right|$ & $\left|x_{3}-\alpha\right|$ & COC \\
\hline M6 & $0.1211(-32)$ & $0.6672(-199)$ & $0.1869(-1196)$ & 6.00 \\
\hline KT6 & $0.4648(-32)$ & $0.4993(-195)$ & $0.7668(-1173)$ & 6.00 \\
\hline ZLH6 & $0.1989(-32)$ & $0.1312(-197)$ & $0.1081(-1188)$ & 6.00 \\
\hline S6 & $0.2659(-32)$ & $0.1001(-196)$ & $0.2851(-1183)$ & 6.00 \\
\hline
\end{tabular}

Table 2: Numerical results for $f_{1}(x), x_{0}=1.36, \alpha \approx 1.36523 \ldots, \gamma_{0}=0.1$

\begin{tabular}{|l|c|c|c|c|}
\hline Methods & $\left|x_{1}-\alpha\right|$ & $\left|x_{2}-\alpha\right|$ & $\left|x_{3}-\alpha\right|$ & COC \\
\hline M6 & $0.4348(-11)$ & $0.1198(-67)$ & $0.5069(-407)$ & 6.00 \\
\hline KT6 & $0.3559(-9)$ & $0.3119(-58)$ & $0.2373(-352)$ & 5.99 \\
\hline ZLH6 & $0.1758(-9)$ & $0.5478(-58)$ & $0.4626(-349)$ & 6.00 \\
\hline S6 & $0.1106(-9)$ & $0.4464(-59)$ & $0.1672(-355)$ & 6.00 \\
\hline
\end{tabular}

Table 3: Numerical results for $f_{2}(x), x_{0}=1.42, \alpha=\sqrt{2}, \gamma_{0}=0.1$

\begin{tabular}{|l|c|c|c|c|}
\hline Methods & $\left|x_{1}-\alpha\right|$ & $\left|x_{2}-\alpha\right|$ & $\left|x_{3}-\alpha\right|$ & COC \\
\hline M6 & $0.1417(-3)$ & $0.5304(-23)$ & $0.1913(-139)$ & 5.99 \\
\hline KT6 & $0.1940(-3)$ & $0.1421(-21)$ & $0.2462(-130)$ & 5.99 \\
\hline ZLH6 & $0.8745(-4)$ & $0.3056(-24)$ & $0.6995(-147)$ & 5.99 \\
\hline S6 & $0.5930(-4)$ & $0.1170(-25)$ & $0.9408(-156)$ & 5.99 \\
\hline
\end{tabular}

Table 4: Numerical results for $f_{3}(x), x_{0}=1.9, \alpha=2, \gamma_{0}=0.1$

developed in Section 3. We will denote this scheme by KT12.

$$
\left\{\begin{array}{l}
x_{0}, w_{0}, \gamma_{0} \text { are given suitably } \\
w_{n}=x_{n}+\gamma_{n} f\left(x_{n}\right) \\
y_{n}=x_{n}-\frac{f\left(x_{n}\right)}{f\left[w_{n}, x_{n}\right]} \\
z_{n}=y_{n}-\frac{f\left(y_{n}\right) f\left(w_{n}\right)}{\left(f\left(w_{n}\right)-f\left(y_{n}\right)\right) f\left[x_{n}, y_{n}\right]} \\
x_{n+1}=z_{n}-\frac{f\left(y_{n}\right) f\left(w_{n}\right)\left(y_{n}-x_{n}+\frac{f\left(x_{n}\right)}{\left(f\left(y_{n}\right)-f\left(z_{n}\right)\right)\left(f\left(w_{n}\right)-f\left(z_{n}\right)\right)}+\frac{f\left(y_{n}\right)}{f\left[y_{n}, z_{n}\right]}\right.}{\gamma_{n+1}=-1 / N_{4}^{\prime}\left(x_{n+1}\right), \quad n=0,1, \ldots}
\end{array}\right.
$$

The corresponding optimal eighth-order method from Zheng et al. (see [27]) gives us the following iterative scheme with memory with order 12, denoted by ZLH12.

$$
\left\{\begin{array}{l}
x_{0}, w_{0}, \gamma_{0} \text { are given suitably, } \\
w_{n}=x_{n}+\gamma_{n} f\left(x_{n}\right) \\
y_{n}=x_{n}-\frac{f\left(x_{n}\right)}{f\left[x_{n}, w_{n}\right]}, \quad f\left(y_{n}\right) \\
z_{n}=y_{n}-\frac{\bar{f}\left[y_{n}, w_{n}\right]+f\left[y_{n}, x_{n}, w_{n}\right]\left(y_{n}-x_{n}\right)}{f\left(z_{n}\right)} \\
x_{n+1}=z_{n}-\frac{\left.z_{n}, y_{n}\right]+f\left[z_{n}, y_{n}, x_{n}\right]\left(z_{n}-y_{n}\right)+f\left[z_{n}, y_{n}, x_{n}, w_{n}\right]\left(z_{n}-y_{n}\right)\left(z_{n}-x_{n}\right)}{f\left[z_{n}\right.}, \\
\gamma_{n+1}=-1 / N_{4}^{\prime}\left(x_{n+1}\right), \quad n=0,1,2, \ldots
\end{array}\right.
$$

Finally, from the optimal eighth-order method proposed by Soleymani et al. in [28] we get the scheme

$$
\left\{\begin{array}{l}
x_{0}, w_{0}, \gamma_{0} \text { are given suitably, } \\
w_{n}=x_{n}+\gamma_{n} f\left(x_{n}\right), \\
y_{n}=x_{n}-\frac{f\left(x_{n}\right)}{f\left[w_{n}, x_{n}\right]}, \\
z_{n}=y_{n}-\frac{f\left(y_{n}\right)}{f\left[y_{n}, x_{n}\right]+f\left[w_{n}, x_{n}, y_{n}\right]\left(y_{n}-x_{n}\right)+\left(y_{n}-x_{n}\right)\left(y_{n}-w_{n}\right)}, \\
x_{n+1}=z_{n}-\frac{f\left(z_{n}\right)}{f\left[x_{n}, z_{n}\right]+M\left(x_{n}-z_{n}\right)+\left(z_{n}-x_{n}\right)\left(z_{n}-w_{n}\right)\left(z_{n}-y_{n}\right)}, \\
\gamma_{n+1}=-1 / N_{4}^{\prime}\left(x_{n+1}\right), \quad n=0,1,2, \ldots,
\end{array}\right.
$$

where $M=f\left[w_{n}, x_{n}, y_{n}\right]-f\left[w_{n}, x_{n}, z_{n}\right]-f\left[y_{n}, x_{n}, z_{n}\right]$, that we denote by $\mathrm{S} 12$. 


\begin{tabular}{|l|c|c|c|c|}
\hline Methods & $\left|x_{1}-\alpha\right|$ & $\left|x_{2}-\alpha\right|$ & $\left|x_{3}-\alpha\right|$ & COC \\
\hline M12 & $0.1940(-65)$ & $0.6241(-792)$ & $0.7676(-9510)$ & 12.00 \\
\hline KT12 & $0.2266(-64)$ & $0.4720(-778)$ & $0.3146(-9342)$ & 12.00 \\
\hline ZLH12 & $0.1940(-65)$ & $0.6241(-792)$ & $.7676(-9510)$ & 12.00 \\
\hline S12 & $0.1685(-65)$ & $0.1000(-792)$ & $0.1918(-9519)$ & 12.00 \\
\hline
\end{tabular}

Table 5: Numerical results for $f_{1}(x), x_{0}=1.36, \alpha=1.36523 \ldots, \gamma_{0}=0.1$

\begin{tabular}{|l|c|c|c|c|}
\hline Methods & $\left|x_{1}-\alpha\right|$ & $\left|x_{2}-\alpha\right|$ & $\left|x_{3}-\alpha\right|$ & COC \\
\hline M12 & $0.9125(-19)$ & $0.2244(-228)$ & $0.1045(-2743)$ & 12.00 \\
\hline KT12 & $0.3976(-18)$ & $0.1410(-220)$ & $0.5304(-2650)$ & 12.00 \\
\hline ZLH12 & $0.9125(-19)$ & $0.2244(-228)$ & $0.1045(-2743)$ & 12.00 \\
\hline S12 & $0.5861(-19)$ & $0.7079(-231)$ & $0.6520(-2774)$ & 12.00 \\
\hline
\end{tabular}

Table 6: Numerical results for $f_{2}(x), x_{0}=1.42, \alpha=\sqrt{2}, \gamma_{0}=0.1$

\begin{tabular}{|l|c|c|c|c|}
\hline Methods & $\left|x_{1}-\alpha\right|$ & $\left|x_{2}-\alpha\right|$ & $\left|x_{3}-\alpha\right|$ & COC \\
\hline M12 & $0.6307(-8)$ & $0.2933(-97)$ & $0.2329(-1169)$ & 12.00 \\
\hline KT12 & $0.7860(-7)$ & $0.2027(-83)$ & $0.1626(-1002)$ & 12.00 \\
\hline ZLH12 & $0.6307(-8)$ & $0.2933(-97)$ & $0.2329(-1169)$ & 12.00 \\
\hline S12 & $0.2124(-9)$ & $0.7767(-113)$ & $0.2441(-1355)$ & 12.01 \\
\hline
\end{tabular}

Table 7: Numerical results for $f_{3}(x), x_{0}=1.9, \alpha=2, \gamma_{0}=0.1$

Tables 5, 6 and 7 present numerical results for the methods with memory pointed out above and M12. It is observed that all these methods behave very well in practice and confirm their theoretical results. They all provide 12th-order of convergence asymptotically without any new functional evaluations.

\section{Conclusions}

We have considered some fourth- and eighth-order schemes from a recent manuscript by Khattri and Steihaug with a parameter in the first step. We have analyzed the complex dynamical behavior of these two classes on quadratic polynomials, getting some conflictive values of the parameter that gives undesirable behavior of the methods and finding others with quite stable performance. As it has been stated previously, it is known and very used that this kind of analysis on quadratic polynomials gives us important information about its stability and reliability of the elements of the class and, as we have said in the introduction, this information can be extrapolated under certain points of view to other more complex functions.

Further on, we have estimated parameter $\gamma$ in each iteration by using the data from the current and previous iterations in such a way that we were able to increase the convergence order from $2^{n}$ to $2^{n}+2^{n-1}$ without any new functional evaluations. Moreover, for illustrating the numerical stability of the developed methods in comparison with their corresponding partners without memory, we have compared the basins of attractions of the original schemes and also of the proposed ones, not only on a quadratic polynomial $p(z)$, but also on a cubic one and on the friction factor problem, concluding the conjecture of "memorizing" the iterative methods implies the widening of areas of convergence and avoids the appearance of attracting strange fixed points. This fact has been empirically checked in the numerical section on the problem of estimating the friction factor of a pipe and also on some academic examples.

Acknowledgments: The authors thank to the anonymous referees for their valuable comments and for the suggestions that have improved the final version of the paper.

\section{References}

1. S. Amat, S. Busquier, C. Bermúdez, S. Plaza, On two families of high order Newton type methods, Applied Mathematics Letters 25 (2012) 2209-2217.

2. S. Amat, S. Busquier, C. Bermúdez, Á.A. Magreñán, On the election of the damped parameter of a two-step relaxed Newtontype method, Nonlinear Dyn. doi:10.1007/s11071-015-2179-x. 
3. C. Chun, B. Neta, An analysis of a family of Maheshwari-based optimal eighth order methods, Applied Mathematics and Computation 253 (2015) 294-307.

4. D.K.R. Babajee, A. Cordero, F. Soleymani, J.R. Torregrosa, On improved three-step schemes with high efficiency index and their dynamics, Numerical Algorithms 65(1) (2014) 153-169.

5. I.K. Argyros, Á.A. Magreñán, On the convergence of an optimal fourth-order family of methods and its dynamics, Applied Mathematics and Computation 252 (2015) 336-346.

6. M. Petković, B. Neta, L. Petković and J. Džunić, Multipoint methods for solving nonlinear equations, Academic Press, 2013.

7. A.M. Ostrowski, Solution of Equations and System of Equations, Prentice-Hall, Englewood Cliffs, NJ, USA, 1964.

8. H.T. Kung, J.F. Traub, Optimal order of one-point and multipoint iteration, J. ACM 21 (1974) 643-651.

9. S.K. Khattri, T. Steihaug, Algorithm for forming derivative-free optimal methods, Numerical Algorithms 65(4) (2014) 809-824.

10. J. F. Traub, Iterative Methods for the Solution of Equations, Prentice Hall, New York, 1964.

11. A. Cordero, F. Soleymani, J.R. Torregrosa, S. Shateyi, Basins of Attraction for Various Steffensen-Type Methods, Journal of Applied Mathematics, Volume 2014, Article ID 539707, 17 pages.

12. R.L. Devaney, The Mandelbrot Set, the Farey Tree and the Fibonacci sequence, American Mathematical Monthly, 106(4) (1999) 289-302.

13. C. McMullen, Families of rational maps and iterative root-finding algorithms, Annals of Mathematics 125(3) (1987) 467-493.

14. F. Chicharro, A. Cordero, J.M. Gutiérrez, J.R. Torregrosa, Complex dynamics of derivative-free methods for nonlinear equations, Applied Mathematics and Computation 219 (2013) 70237035.

15. Á. A. Magreñán, Different anomalies in a Jarratt family of iterative root-finding methods, Applied Mathematics and Computation 233 (2014) 29-38.

16. B. Neta, C. Chun, M. Scott, Basins of attraction for optimal eighth order methods to find simple roots of nonlinear equations, Applied Mathematics and Computation 227 (2014) 567-592.

17. T. Lotfi, Á.A. Magreñán, K. Mahdiani, J.J. Rainer, A variant of Steffensen-King's type family with accelerated sixth-order convergence and high efficiency index: Dynamic study and approach, Applied Mathematics and Computation 252 (2015) 347353.

18. F.I. Chicharro, A. Cordero, J.R. Torregrosa, Drawing dynamical and parameters planes of iterative families and methods, The Scientific World Journal Volume 2013, Article ID 780153, 11 pages.

19. A. Cordero, T. Lotfi, J.R. Torregrosa, P. Assari, K. Mahdiani, Some new bi-accelerator two-point methods for solving nonlinear equations, Computational and Applied Mathematics 35(1) (2016) 251-267.

20. A. Cordero, T. Lotfi, P. Bakhtiari, J.R. Torregrosa, An efficient two-parametric family with memory for nonlinear equations, Numerical Algorithms 68(2) (2015) 323-335.

21. T. Lotfi, K. Mahdiani, P. Bakhtiari, F. Soleymani, Constructing two-step iterative methods with and without memory, Computational Mathematics and Mathematical Physics 55(2) (2015) 183-193.

22. A. Cordero, J. G. Maimó, J.R. Torregrosa, M. P. Vassileva, Solving nonlinear problems by Ostrowski-Chun type parametric families, J. Math. Chem. 53 430-449 (2015)

23. M. Abad, A. Cordero, J.R. Torregrosa, A family of seventh-order schemes for solving nonlinear systems, Bull. Math. Soc. Sci. Math. Roumanie Tome 57(105)(2014) 133-145.

24. S. Weerakoon, T.G.I. Fernando, A variant of Newton's method with accelerated third-order convergence, Apllied Mathematics Letters 13 (2000) 87-93.

25. F. White, Fluid Mechanics, McGraw-Hill, Boston 2003.

26. H.T. Kung, J.F. Traub, Optimal order of one-point and multipoint iteration, J. ACM 21 (1974) 643-651.

27. Q. Zheng, J. Li, F. Huang, An optimal Steffensen-type family for solving nonlinear equations, Appl. Math. Comput. 217 (2011) 9592-9597.

28. F. Soleymani, D.K R. Babajee, S. Shateyi, S.S. Motsa, Construction of Optimal Derivative-Free Techniques without Memory, Journal of Applied Mathematics Volume 2012, Article ID 497023, 24 pages doi:10.1155/2012/497023. 